\title{
Combined design of structures and controllers for optimal maneuverability
}

\author{
J. Ling, P. Kabamba and J. Taylor \\ Department of Aerospace Engineering, The University of Michigan, Ann Arbor, MI 48109-2140, USA
}

\begin{abstract}
This paper treats the problem of the combined design of structure/control systems for achieving optimal maneuverability. A maneuverability index which directly reflects the time required to perform a given maneuver or set of maneuvers is introduced. By designing the flexible appendages of a spacecraft, its maneuverability is optimized under the constraints of structural properties, and of the postmaneuver spill-over being within a specified bound. The spillover reduction is achieved by making use of an appropriate control design model. The distributed parameter design problem is approached using assumed shape functions and finite element analysis with dynamic reduction. Characteristics of the problem and problem solving procedures have been investigated. Adaptive approximate design methods have been developed to overcome computational difficulties. It is shown that the global optimal design may be obtained by tuning the natural frequencies of the spacecraft to satisfy specific constraints. We quantify the difference between a lower bound to the objective function associated with the original problem and the estimate obtained from the modified problem as the index for the adaptive refinement procedure. Numerical examples show that the results of the optimal design can provide substantial improvement.
\end{abstract}

\section{Introduction}

Large space structures such as antennas or space stations will be very flexible, not only because of the high cost of transportation of structures from earth to space, but also because they will be constructed or deployed in orbit and will not need to withstand large launching and gravity loads. However, when a space structure is very flexible, its active control system can excite and otherwise significantly interact with its flexible modes. Thus, the idea arises of achieving simultaneous vibration mode suppression with attitude maneuver of flexible spacecraft. The control design for rotational maneuvers and vibration mode suppression of flexible spacecraft has received extensive attention (e.g. Breakwell 1981; Van der Velde and He 1983; Turner and Chun 1984; Hale and Lisowski 1985; Vadali 1986; Fujii and Ishijima 1989; BenAsher et al. 1987; Barbieri and Ozguner 1988; Thompson et al. 1989, 1990; Singh et al. 1989). For certain applications, it will be desirable that such a spacecraft slew as rapidly as possible, within the operating limits of the control actuators. Flexible spacecraft are modelled with a finite number of vibration modes, and optimal control theory is then applied to obtain the time optimal control history for quiescent terminal conditions of the vibration modes (see, for example, Barbieri and Ozguner 1988; Thompson et al. 1989, 1990; Singh et al. 1989). Singh et al. (1989) observe quantitatively that the difference between the minimum time for a rigid body and the actual time required for a flexible spacecraft is small for large-angle maneuvers of systems of low flexibility and low authority control torques. However, all of these authors only consider control design based on a specific structure. It is expected that by adjusting the design parameters of the spacecraft itself we may obtain even better results, especially for large flexible structures. As a consequence, structural optimization is considered so as to further minimize the maneuver time and achieve vibration mode suppression. The design process is thus a combined design of controllers/structures applied to spacecraft. This paper is concerned with developing a theoretical and practical framework for solving this problem.

Traditionally, the overall design for actively controlled space structures is treated via an iterative two-part scheme. The redesign of the structure including sensor and actuator placement is performed in one stage, and then the control law is modified for the resulting system to complete an iteration cycle. Generally different design objectives apply in the separate steps. More recently, the need to integrate the design of a structure and its control system has been recognized. An integrated approach is justified simply on the basis that structural and control purposes are substantially coupled. Bodden and Junkins (1985) presented a method for eigenvalue optimization with sequential or simultaneous design of structure and control. Khot et al. (1985a, b) and Khot et al. (1988) considered structural optimization, including constraints on control gain norm and transient behaviour of the control system, based on a linear-quadratic model of the controller. Hale and Lisowski (1983) and Hale et al. (1984) treated the problem of simultaneous structure and control design for a maneuvering spacecraft which resulted in a linear-quadratic optimization problem. Bendsøe et al. (1987) presented an algorithm for integrated design of the structure and its control system which includes a constraint to limit the controller spillover from the unmodelled modes. Lust and Schmit (1988) presented a control-augmented structural synthesis methodology in which the structural member sizes and active control system feedback gains are treated simultaneously as independent design variables. Onoda and Haftka (1987) and Haftka et al. (1985) considered the optimization of the total cost of the structure and control system subject to constraints on the magnitude of the response to a given disturbance involving both rigid-body and elastic modes. Lim and Junkins 
(1989) presented an idea for optimizing the robustness of the controlled structures, using homotopy and sequential linear programming algorithms. Khot (1988) presented algorithms for the design of minimum weight structures with the goal of improving system dynamics by use of a closed-loop control system.

Most of the developments on simultaneous design of structures and controllers reported in the literature use simple linear feedback control laws and quadratic performance indices. Practical constraints such as limitation on the amplitude of the control effort are, in general, not taken into account. The use of such relatively narrow forms of problem statements may have serious implications in terms of the usefulness of the results. It is understood, for example, that the use of performance indices expressed as linear/quadratic functionals is generally inappropriate unless loop transfer recovery techniques (see, for example, Safonov and Athans 1977; Lehtomaki et al. 1981; Doyle 1987; Doyle and Stein 1979) are incorporated into the formulation. Furthermore, in the literature, the constraints usually used are on the closed-loop eigenvalue distribution and structural frequencies. These constraints are not as direct to the application problem as constraints on rise time, maximal displacement, or maximal stress. The consideration of performance degradation of the optimal system coming from the control and observer spillover is also generally not included.

In the present work, the problem of fully coupled design for a spacecraft and its associated control is examined. The design of the structural system and control is to be integrated so as to optimize with respect to a single cost function. The objective is chosen to reflect the maneuverability of this structure/control system, i.e. the time required to perform a given maneuver or set of maneuvers. Various forms of mission specification can be reflected in the definition of the maneuverability index. It includes criteria related to sets of maneuvers with specified probability of occurence. This maneuverability index is generally more meaningful than the usual LQG index with minimum weight. The 'minimum time' objective is appropriate for application in slewing or other retargeting maneuvers. Furthermore, the problem is formulated in a way to accommodate in explicit form various practical constraints, such as limits on control action and performance error (control spillover). Also, the formulation is consistent with a nonlinear bang-bang form of optimal control design.

The spacecraft is modelled as a linear, elastic, undamped, nongyroscopic system. The control design is based on a reduced order model which is appropriately chosen with control spillover taken into account. The dynamic properties of the reduced order model are obtained by the finite element and dynamic reduction methods. The necessary-andsufficient conditions for the time-optimal rest-to-rest control problem, obtained by Singh et al. (1989), are considered as a mapping from the structural dynamic properties to the optimal maneuver time. For the design of the spacecraft we assume that the maneuverability index is optimized so as to reflect the optimal maneuver time of a given maneuver or set of maneuvers. The maneuverability index is optimized by updating structural design parameters. Characteristics of the problem and problem solving procedures have been investigated. Approximate design methods have been developed to overcome computational difficulties. Numerical examples are presented to demonstrate the capability of this approach.

\section{Combined design of structures/controllers - problem statement}

Flexible spacecraft are usually modelled as systems of interconnected rigid bodies and flexible appendages. Consider the linearized rotational dynamics of a flexible spacecraft where control inputs are used to actively control the rigid body mode and flexible modes. The spacecraft consists of a cylindrical symmetric rigid central body, as shown in Fig. 1, to which $N(N \geq 2)$ identical flexible appendages are attached with uniform spacing between them. Along the appendages, there may be distributed or concentrated payload masses, and the spacecraft may be very large and flexible. It is to be controlled by a single torque actuator located on the central body and $m$ torquers located at identical locations on each of the $N$ appendages. The amplitude of the torque applied by each torquer is limited. The extension of the problem formulation to a system controlled by force actuators or a combination of torquer and force actuators is straight-forward and yields similar dynamical equations. In the subsequent analysis, large rotations of the rigid central body are considered and the specific vehicle geometry and actuator locations assure that the translational motion of the vehicle centre of mass and the rotational motion of the vehicle are decoupled. A more general vehicle geometry, where $N(N \geq 2)$ flexible appendages, not necessarily identical, are arbitrarily attached to the rigid central body, can be considered if the analysis is restricted to cases where translation and rotation of the rigid central body are "small", reflecting small structural deformations. Situations where only one appendage is attached to the rigid central body can also be considered provided the rigid central body is constrained against translation.

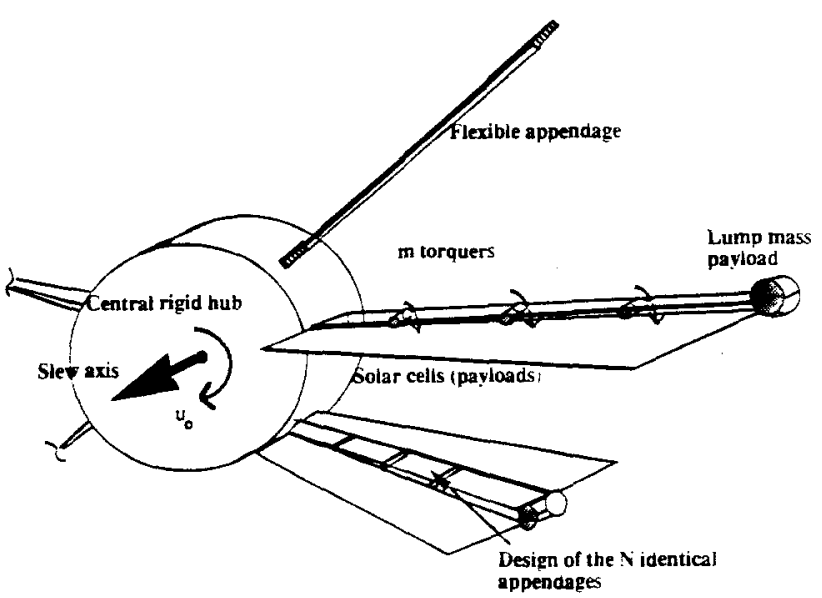

Fig. 1. The generic flexible spacecraft model

The following assumptions are made to obtain a mathematical model of the system:

1. the appendage displacements and slopes are small relative to their undeformed shapes;

2. the appendage shear deformations are negligible compared to the bending deformations; 
3. the appendages are inextensible;

4. appendage displacements are restricted to a plane orthogonal to the central body's axis of rotation;

5. no structural damping is present;

6 . the deformation of the appendages relative to their undeformed shapes are identical and antisymmetric;

7. the rigid central body rotation rate remains small at all times;

8. the spacecraft centre of mass coincides with that of the rigid central body;

9. the spacecraft centre of mass does not translate in inertial space.

The design parameters of the appendages can be crosssection, stiffness or density of the material, layout of the composite material or the location of torque actuators along the appendage. Let the design parameter vector be $\xi \in R^{N}$, implying that the structural dynamic properties are implicit functions of $\xi$.

\subsection{Maneuverability index}

The maneuverability index reflects the time required to perform a given maneuver or set of maneuvers. The mission profile is specified by giving the probability density function $p(\theta)$ of the required maneuver amplitude $\theta$. For the design of the spacecraft we assume that the maneuverability index is optimized so as to reflect the optimal maneuver time of a given maneuver or set of maneuvers. Let $t_{f}^{*}(\theta)$ be the optimal maneuver time for a rest-to-rest maneuver of angles $\theta$, so that $t_{f}^{*}(\theta)$ is an implicit function of the structural design parameter vector $\xi$. Therefore, the maneuverability index is also a function of $\xi$, defined as

$\mu^{*}(\xi)=\int_{-\infty}^{+\infty} p(\theta) \cdot t_{f}^{*}(\theta) \mathrm{d} \theta$

For example, let $p(\theta)=\delta\left(\theta-\theta^{*}\right)$ then $\mu^{*}(\xi)=t_{f}^{*}\left(\theta^{*}\right)$. In other words, the maneuverability index represents the expected value of the optimal maneuver time for a given mission profile. The structural design problem is then to optimize $\mu^{*}(\xi)$ with respect to $\xi$.

\subsection{Optimal design problems}

Assume that the structural design parameter vector $\xi$ is restricted to belong to a compact set $\Xi$, which represents feasible designs. Assume that the design of the appendages will not change the characteristics of the torquers along the appendages. In other words, the amplitude limits of the torquers remain the same for all values of the design parameters. Therefore, we can formulate the optimal combined structure/control design problem as

$\mu=\min \mu^{*}(\xi), \quad \xi \in \Xi$,

where $\Xi$ is the space of structural design variables, subject to two sets of constraints:
I a material resource constraint;

b geometric configuration constraints: such as max and min thickness limits of cross-section;

c dynamic response constraints: such as max stress and displacement limits;

and

II the postmaneuver control spillover is within a specified bound.

Constraint II takes into account the perfomance degradation associated with the unmodelled dynamics.

We approach the distributed parameter design of the cross-section of the appendage using assumed shape functions. More specifically, let the design parameter of the cross-section be the thickness distribution. We assume that the thickness function, $h(x)$ ( $x$ is the location along the appendage), is a linear combination of known shape functions, where the coefficients are design variables to be optimized. This approach uses the same idea as design variable linkage (e.g. Fetterman and Noor 1987).

3 Formulation and characteristics of the optimal design problem

\subsection{Equations of motion}

Let $\theta(t)$ be the angular displacement of the rigid central body, and $y(x, t)$ be the lateral displacement of the flexible appendages at $x$, the distance along the appendage measured from the appendage root, $0 \leq x \leq L$; where $L$ is the overall length of an appendage. Let $Q_{\theta}$ and $Q_{y}$ be the generalized forces associated with $\theta(t)$ and $y$, respectively. Let $J^{*}$ be the total rotational inertia of the undeformed vehicle about the axis of rotation. Let the central body be cylindrical with radius $R$ and total mass $m_{0}, E I(x)$ be the appendage material stiffness, $\rho(x)$ be the mass distribution of the appendage per unit length (including the payload mass). Here boldface type is used to denote matrices and vectors; \{\}$_{x},\{\}_{x x}$ denote first and second partial derivatives, respectively, and [ $]^{t}$ denotes transpose.

The application of Hamilton's principle results in the following coupled linear partial and ordinary differential equations and associated boundary conditions:

$$
J^{*}=1 / 2 m_{0} R^{2}+N \int_{0}^{L} \rho(x) \cdot(R+x)^{2} \mathrm{~d} x,
$$

and

$$
\begin{aligned}
& J^{*} \ddot{\theta}+N \int_{0}^{L} \rho(x)(R+x) y_{t t} \mathrm{~d} x=Q_{\theta}(t), \\
& N\left[\left\{E I(x) y(x, t)_{x x}\right\}_{x x}+\rho(x)\left\{y(x, t)_{t t}+(R+x)\right\} \ddot{\theta}\right]= \\
& =Q_{y}(t, x),
\end{aligned}
$$$$
\left[\left\{E I(x) y_{x x}\right\}_{x} \delta y\right]_{[x=L]}-\left[\left\{E I(x) y_{x x}\right\}_{x} \delta y\right]_{[x=0]}=0,
$$ 
$\left[E I(x) y_{x x} \delta y_{x}\right]_{[x=L]}-\left[E I(x) y_{x x} \delta y_{x}\right]_{[x=0]}=0$,

where $Q_{y}$ and $Q_{\theta}$ are the generalized forces.

The "exact" solution of this system generally consists of infinite sets of eigenfunctions and associated eigenvalues (e.g. Meirovitch 1976). It can be approximated with the assumed mode method, i.e. $y(x, t)$ can be expressed as

$y(x, t)=\sum_{i=1}^{\infty} \phi_{i} \cdot q_{i}$

where $\phi_{i}(x)$ and $q_{i}(t)$ are complete assumed mode shapes and the corresponding generalized coordinates, respectively.

We use the finite element method, whereby appendages of the spacecraft are discretized into a finite number of beam elements. Therefore, the number of terms in (4) is finite and $\phi_{i}(x)$ are the elemental Hermite cubics and $q_{i}(t)$ are the nodal degrees of freedom.

We use two mathematical models for design and analysis, the control design model and the control evaluation model. The number of modes in the control evaluation model is the number of degrees of freedom in the finite element analysis (let it be $n$ herein). Assuming this model to represent the exact dynamic system, we evaluate the performance of the controller on it. The control design is the model from which we obtain the time-optimal maneuver law. We assume there are $r(r \ll n)$ vibrational modes retained in this model. In other words the $(r+1)$-th to $n$-th modes are uncontrolled.

With the finite element method, (3) results in the following coupled linear ordinary differential equations:

$J^{*} \ddot{\theta}+\mathbf{m}^{\mathbf{t}} \ddot{\mathbf{q}}=Q_{\theta}, \quad \mathbf{M} \ddot{\mathbf{q}}+\mathbf{K} \mathbf{q}+\mathbf{m} \ddot{\theta}=\left[\mathbf{Q}_{\mathbf{y}}(\mathbf{t})\right]$,

where the elements of the $n \times n$ matrices $\mathrm{M}, \mathrm{K}$ and the $n \times 1$ vector $\mathbf{m}$ are

$$
\left\{\begin{aligned}
m_{i} & \equiv N \int_{0}^{L} \rho(x)(R+x) \phi_{i} \mathrm{~d} x, \\
M_{i j} & \equiv N \int_{0}^{L} \rho(x) \phi_{i} \phi_{j} \mathrm{~d} x, \\
K_{i j} & \equiv N \int_{0}^{L}\left\{\phi_{i}\right\}\left[E I(x)\left\{\phi_{j}\right\}_{, x x}\right], x x \mathrm{~d} x= \\
& =N \int_{0}^{L} E I(x)\left\{\phi_{i}\right\}_{, x x}\left\{\phi_{j}\right\}_{, x x} \mathrm{~d} x,
\end{aligned}\right.
$$

where

$i=1,2, \ldots, n, \quad j=1,2, \ldots, n, \quad$ and

$q=\left[q_{1}(t), q_{2}(t), q_{3}(t), \ldots, q_{n}(t)\right]^{t}$.

Let $u_{0}(t)$ be the torque applied by the torquer located on the rigid central body and $u_{j}(t), j=1,2, \ldots, m$, be the total torque, applied by the $N$ torquers located at $x=L_{j}$ along each appendage (see Fig. 1). It is easily shown that the generalized forces are

$Q_{\theta}=u_{0}(t)+N \sum_{1}^{m} u_{j}(t)$,
$\left.\left[\mathbf{Q}_{\mathbf{y}}(\mathbf{t})\right]_{i}=N \sum_{j=1}^{m}\left[\left\{\phi_{i}(x)\right\}_{x}\right]_{\left[x=L_{j}\right.}\right]_{j}(t)$

We eliminate the first equation of (5) and introduce a coordinate transformation $q=V \eta$, where $V$ is a normalized modal matrix such that

$\mathbf{V}^{\mathbf{t}}\left[\mathbf{M}-\left(1 / J^{*}\right) \mathbf{m m}^{\mathbf{t}}\right] \mathbf{V}=\mathbf{I}$ and $\mathbf{V}^{\mathbf{t}} \mathbf{K V}=\boldsymbol{\Omega}^{2}$,

where $I$ is a unit matrix and $\boldsymbol{\Omega}^{2}=\operatorname{diag}\left(\omega_{i}^{2}\right)$, $i=1,2,3, \ldots, n$.

Then (5) is transformed into

$\dot{\boldsymbol{\eta}}+\boldsymbol{\Omega}^{2} \boldsymbol{\eta}=\mathbf{V}^{t}\left\{\left[\mathbf{Q}_{\mathbf{y}}(\mathbf{t})\right]-Q_{\theta}(t) / J^{*} \mathbf{m}\right\}$.

\subsection{Results on linear time-optimal control problems}

Results presented in this section were reported in a recent paper by Singh et al. (1989). The optimal control characterized here is based on a control design model. Assume that there are $r$ flexible modes in the control design model. They are the first $r$ flexible modes of the $n$ modes of (9).

Define the state vector as $\mathbf{x}=\left(x_{1}, x_{2}, x_{3}^{1}, x_{4}^{1}, \ldots, x_{3}^{r}\right.$, $\left.x_{4}^{r}\right)^{t}$ and the control vector as $\mathbf{u}=\left(u_{0}, u_{1}, \ldots, u_{m}\right)^{t}$, where

$x_{1}=\theta+\left(1 / J^{*}\right) \mathbf{m}^{\mathbf{t}} \mathbf{q}, \quad x_{2}=\dot{x}_{1}, \quad x_{3}^{i}=\eta_{i}$,

$x_{4}^{i}=\dot{\eta}_{i} / \omega_{i}, \quad i=1,2,3, \ldots, r, \quad j=1,2, \ldots, m$.

Define the modal control influence parameters

$\beta_{0}^{0}=1 / J^{*}, \quad \beta_{j}^{0}=N / J^{*}$,

$\beta_{0}^{i}=-\left(1 / J^{*}\right) / \omega_{i}\{v\}_{i}^{t} \mathbf{m}$,

$\beta_{j}^{i}=N / \omega_{i}\left(\{v\}_{i}^{t}\left[\left\{\phi_{i}(x)\right\}_{x}\right]_{\left[x=L_{j}\right]}-\left(1 / J^{*}\right)\{v\}_{i}^{t} \mathbf{m}\right)$,

$i=1,2, \ldots, r ; \quad j=1,2, \ldots, m$,

where $\omega_{i}$ is the $i$-th natural frequency and $\{v\}_{i}$ is the eigenvector corresponding to $\omega_{i}$. In the finite element analysis, we discretize the appendage in such a way that there is always a node at the location of each actuator, $x=L_{j}$. Then $\left[\left\{\phi_{i}\right\}_{x}\right]_{\left[x=L_{j}\right]}$ of (7) is equal to one. The natural frequencies and eigenvectors of the first $r$ modes are obtained from the full matrices of (8) with the dynamic reduction method (see, e.g. Fetterman and Noor 1987). All $\beta_{0}^{i}, \beta_{j}^{i}, i=1,2, \ldots, r ; j=1,2, \ldots, m$, are then obtained from the $r$ eigenvectors.

Let subscript $c$ indicate that a quantity is obtained based on the control design model. The problem of time-optimal rest-to-rest slewing maneuver can be formulated as follows.

Problem $\mathrm{M}(\theta)_{\mathcal{C}}$ :

$\min t_{f}(\theta)_{\mathcal{C}}$ 
subject to

$\dot{\mathbf{x}}=\mathbf{A x}(\mathbf{t})+\mathbf{B u}(\mathbf{t}), \quad\left|u_{j}(t)\right| \leq U_{j}, \quad j=0,1, \ldots, m$,

where $U_{j}, j=0,1, \ldots, m$ are the corresponding amplitude limits of $u_{j}$,

$\mathbf{x}(0)=(0,0, \ldots, 0)^{t}, \quad \mathbf{x}\left(t_{f}\right)=(\theta, 0, \ldots, 0)^{t}$

$\mathbf{A}=$ Block diag $\left[\mathbf{A}_{i}\right], \mathbf{B}=$ Block col $\left[\mathbf{B}_{i}\right]$, where

$\mathbf{A}_{i}=\left\{\begin{array}{l}{\left[\begin{array}{ll}0 & 1 \\ 0 & 0\end{array}\right], \quad i=0,} \\ {\left[\begin{array}{cc}0 & \omega_{i} \\ -\omega_{i} & 0\end{array}\right], \quad i=1,2, \ldots, r}\end{array}\right.$

where $\omega_{i}$ is the natural frequency,

$\mathbf{B}_{i}=\left[\begin{array}{cccc}0 & 0 & \ldots & 0 \\ \beta_{0}^{i} & \beta_{1}^{i} & \ldots & \beta_{m}^{i}\end{array}\right], \quad i=0,1,2, \ldots, r$

Let the solution for the problem $M(\theta)_{\mathcal{C}}$ be $t_{f}^{*}(\theta)$.

Theorem 3.2.1. For all $\theta$, the problem $M(\theta)_{\mathcal{C}}$ has a unique solution $t_{f}^{*}$.

Theorem 3.2.2. For a given $\theta$, the optimal control law of the problem $M(\theta)_{\mathcal{C}}$ is of bang-bang type and is anti-symmetric around $t_{f}^{*} / 2$, i.e. $\mathbf{u}\left(t_{f}^{*} / 2-t\right)^{*}=-\mathbf{u}\left(t_{f}^{*} / 2+t\right)^{*}, 0 \leq t \leq t_{f}^{*} / 2$.

Singh et al. (1989) treat the general multiple control case where there are $m+1$ control inputs. However, for simplicity, herein we assume that only one control input is used to control the maneuver, that is, the scalar control case. This assumption means that the $m$ torque actuators on the appendages and the actuator on the rigid central body taken together represent one control input. Herein, consider a spacecraft controlled by only one torquer located on the rigid central body as a special scalar control case.

Theorem 3.2.3. Assume there are $k$ switching times between 0 and $t_{f}^{*} / 2$, and let them be $t_{i}, i=1,2, \ldots, k$. Let $J^{*}$ be the total rotational moment of the spacecraft, and $\left(p_{0}^{0}, 0, p_{0}^{1}, 0, \ldots, p_{0}^{r}, 0\right)$ be the costate variable at midmaneuver time. Then the optimal maneuver time and the switching times satisfy, as necessary and sufficient conditions, the following system of nonlinear algebraic equations:

$\left(t_{f}^{*} / 2\right)^{2}-2\left(t_{k}\right)^{2}+2\left(t_{k-1}\right)^{2}-\ldots+2(-1)^{k}\left(t_{1}\right)^{2}=$

$\theta J^{*} / U_{0}$

$\cos \left(\omega_{i} t_{f}^{*} / 2\right)-2 \cos \left(\omega_{i} t_{k}\right)+2 \cos \left(\omega_{i} t_{k-1}\right)-\ldots+$

$+2(-1)^{k} \cos \left(\omega_{i} t_{1}\right)+(-1)^{k+1}=0$,

$i=1,2, \ldots, r$,

$\left[\begin{array}{cccc}2 t_{f}^{*} / 2 & 2 \sin \left(\omega_{i} t_{f}^{*} / 2\right) & \ldots & 2 \sin \left(\omega_{r} t_{f}^{*} / 2\right) \\ -4 t_{k} & -4 \sin \left(\omega_{1} t_{k}\right) & \ldots & -4 \sin \left(\omega_{r} t_{k}\right) \\ \vdots & \vdots & & \vdots \\ 4(-1)^{k} t_{1} & 4(-1)^{k} \sin \left(\omega_{1} t_{1}\right) & \ldots & 4(-1)^{k} \sin \left(\omega_{r} t_{1}\right)\end{array}\right]$ $\left\{\begin{array}{c}p_{0}^{0} \\ \beta_{0}^{1} p_{0}^{1} \\ \vdots \\ \beta_{0}^{r} p_{0}^{r}\end{array}\right\}=\left\{\begin{array}{c}-2 / U_{0} \\ 0 \\ \vdots \\ 0 \\ 0\end{array}\right\}$

and two inequality equations

$t_{f}^{*} / 2>t_{k}>t_{k-1}>\ldots>t_{2}>t_{1}>0$,

$p_{0}^{0} t+\sum_{i=1}^{r} \beta_{j}^{i} p_{0}^{i} \sin \left(\omega_{i}^{t}\right) \neq 0$

where

$0<t<t_{f}^{*} / 2, \quad t \neq t_{i}, \quad i=1,2, \ldots, k$.

Corollary 3.2.4. Let there be $k$ switching times between 0 and $t_{f}^{*} / 2$ of the optimal control history for the control design model with $r$ flexible modes. Let the switching times be $t_{j}$, $j=1,2, \ldots k$, and the optimal maneuver time be $t_{f}^{*}$. Suppose that $k=r$. Then the optimal maneuver time and the switching times satisfy as necessary and sufficient conditions

(i) (14), (15) admit a solution for $\left\{t_{j}, j=1,2, \ldots, k\right.$ and $\left.t_{f}^{*}\right\}$ which is regular;

(ii) $\left\{t_{j}, j=1,2, \ldots\right.$ and $\left.t_{f}^{*}\right\}$ satisfy (16) and the inequalties (17).

Proof. The proof of sufficiency can be found in a paper by Singh et al. (1989) and that of necessity in the Appendix.

To solve for the optimal control history, we first need to assume the number of switching times, say $k$, then try to find solutions $\left\{t_{j}, j=1,2, \ldots, k\right\}$ and $\left\{p_{0}^{j}=0, i=\right.$ $0,1,2, \ldots, r\}$ for (14)-(16). If (14)-(16) do admit solutions and they satisfy (17) as well, by the uniqueness of the solution of the optimal control problem, we have the unique solution. We use the homotopy method to solve the system of nonlinear equations (see, e.g. Garcia and Zangwill 1981). We have found that, in general, $k$ is always equal to $r$. Only when $\left\{\omega_{i}, i=1,2, \ldots, r\right\}$ satisfy some special conditions is $k$ less than $r$. For the generic ease where $k$ is equal to $r$, we can use Corollary 3.3.1 to simplify the solving procedure. We only need to solve for $\left\{t_{j}, j=1,2, \ldots, k\right.$ and $\left.t_{f}^{*}\right\}$ from (14) and (15), a small set of the nonlinear equations, and then check the solution with respect to the condition (ii) of Corollary 3.3.1. However the Jacobian of (14) and (15) tends to be singular when two switching times are almost identical or one tends to zero, which implies the number of switches, $k$, is to be decreased and $k$ will be less than $r$. The homotopy method fails to find the solution we want in such ill-conditioned situations. The following is an important corollary, which sheds light on the degenerate case, $k<r$.

Corollary 9.2.5. Consider a control design model with $r$ flexible modes. Suppose that the number of switching times of the optimal control history between 0 and $t_{f}^{*} / 2$ is $k$, where $r>k$. Then a set of sufficient conditions for optimality are as follows: 
(i) (14) and the first $k$ equations of (15) admit a solution $\left\{t_{j}, j=1,2, \ldots, k\right.$ and $\left.t_{f}^{*}\right\}$, which is regular;

(ii) $\cos \left(\omega_{i} t_{f}^{*} / 2\right)-2 \cos \left(\omega_{i} t_{k}\right)+2 \cos \left(\omega_{i} t_{k-1}\right)-\ldots+$ $2(-1)^{k} \cos \left(\omega_{i} t_{1}\right)+(-1)^{k+1}=0$, where $i=k+1, k+$ $2, \ldots, r$; .

(iii) $\left\{t_{j}, j=1,2, \ldots, k\right.$ and $\left.t_{f}^{*}\right\}$ satisfy (16) and inequalties (17), with $\left\{p_{0}^{i}=0, i=k+1, k+2, \ldots, r\right\}$.

Proof. See Appendix.

We can explain the result of Corollary 3.3.2 as the optimal control for a control design model with $k(k<r)$ flexible modes, where, as usual, there are $k$ switches of the control history within $\left(0, t_{f}^{*} / 2\right)$; however, it happens that the $k+$ 1-th to $r$-th flexible modes of the spacecraft are also dead beat with this control. This is an exceptional case and from numerical study we observe that the design of spacecraft for which the optimal control with $k<r$ is an isolated point in the design variable space. Later we will use the result of Corollary 3.2.5 to derive a useful algorithm to solve the time optimal control problem.

\subsection{The control design model}

We now consider how many flexible modes should be retained in the control design model. The question is answered by analysing the degradation in the performance of the controller on the control evaluation model. This performance degradation is associated with the "unmodelled dynamics" of the uncontrolled residual modes in the control evaluation model (from order $r+1$ to order $n$ ). These unmodelled dynamics result in postmaneuver free vibration of the system, due to control spillover. We need to make sure these vibrations have amplitudes within a specified performance error bound during the optimization.

There are two ways to quantify the performance degradation: (i) the residual or spillover energy $\mathcal{E}_{r}$, and (ii) the pointing error of the rigid central body after completion of the maneuver $\theta_{e}(t)$ (where $t \geq t_{f}^{*} / 2$ ). According to a recent investigation of these (Singh et al. 1989), the latter is the better one because the maximum pointing error continues to decrease as we suppress additional modes at the final time, while the spillover energy does not necessarily decrease. Moreover, Singh et al. (1989) give three closed form expressions for an upper bound $\left|\theta_{e}(t)\right|$, based on the control evaluation model. Among them, the most useful according to our experience is

$\left|\theta_{e}(t)\right| \leq 2\left\{(2+2 k) U_{0}\right\} \sum_{i=r+1}^{n}\left(\beta_{0}^{i}\right)^{2}, \quad t \geq t_{f}^{*} / 2$.

We use this upper bound to determine the size of the control design model in order to obtain a prespecified post-maneuver pointing accuracy of the rigid central body. As shown in Table 1 , the upper bound generally decreases dramatically fast as we suppress additional modes. In practical examples with realistic scale and material, one is likely to obtain acceptable control spillover by only suppressing very few modes.
Table 1. Spillover vs. the number of flexible modes in the control design model for a design of spacecraft

\begin{tabular}{|c|c|}
\hline $\begin{array}{c}\text { Number of modes } \\
\text { retained in the } \\
\text { model }\end{array}$ & $\begin{array}{c}\text { Max. angle deviation } \\
\text { post maneuver (deg.) }\end{array}$ \\
\hline 0 & 1.495 \\
1 & 0.082 \\
2 & 0.0133 \\
3 & $2.6 \mathrm{e}-3$ \\
4 & $8.3 \mathrm{e}-4$ \\
5 & $3.2 \mathrm{e}-4$ \\
6 & $1.5 \mathrm{e}-4$ \\
7 & $7.1 \mathrm{e}-5$ \\
\hline
\end{tabular}

\subsection{Characteristics of the optimal design problem}

Theorem 3.4.1. Suppose the number of flexible modes retained in the model is fixed. The optimal maneuver time solved from (14)-(17) is a continuous function of the structural design variable vector $\xi$.

Proof. See Appendix.

Corollary 3.4.2. The objective function, $\mu(\xi)$, is a continuous function of $\xi$.

Corollary 3.4.9. There exists a solution to the optimal design problem (2).

Theorem 3.4.4. Consider the general design cases such that the structural properties are analytical functions of the design parameters. Suppose the number of flexible modes retained in the model is fixed, say $r$. Assume that in a closed region of the feasible design variable space, the number of switching times, say $k$, follows the generic rule, where $k=r$. Then the optimal maneuver time $t_{f}^{*}$ is an analytical function of the structural design variables, $\xi$, in this region.

Proof. See Appendix.

We have observed that the objective function is always a differentiable function of the structural design variables, $\xi$, even when some switches of the control history tend to zero. Consider the generic case where $k$ is equal to $r$. The optimal maneuver time can be obtained from (14) and (15) only (Corollary 3.2.4). Actually (14) and (15) represent a system of implicit equations of the form

$\mathbf{F}\left(t_{f}^{*}, \mathbf{t}_{\mathbf{i}}, \omega, J^{*}\right)=0$,

where $\mathbf{t}_{\mathbf{i}}=\left[t_{1}, t_{2}, \ldots, t_{k}\right]^{t}$ is the vector of switching times, and $\omega=\left[\omega_{1}, \omega_{2}, \ldots, \omega_{r}\right]$ is the vector of natural frequencies.

The gradient of the optimal maneuver time with respect to structural parameters can be obtained using the implicit function theorem as follows: let $\mathbf{x}=\left(t_{f}^{*}, \mathbf{t}_{\mathbf{i}}\right)$ and $\mathbf{y}=\left(\omega, J^{*}\right)$. Theorem 9.4.5 (Implicit function theorem) (Hartman 1982). Let $\mathbf{x} \varepsilon \mathbf{R}^{n}, \mathbf{y} \varepsilon \mathbf{R}^{m}$ and $\mathbf{F}: \mathbf{R}^{n+m} \rightarrow \mathbf{R}^{n}:(\mathbf{x}, \mathbf{y}) \rightarrow \mathbf{F}(\mathbf{x}, \mathbf{y})$. Suppose $\left(\mathbf{x}_{0}, \mathbf{y}_{0}\right)$ is such that $\mathbf{F}\left(\mathbf{x}_{0}, \mathbf{y}_{0}\right)=0$ and $\mathbf{F}\left(\mathbf{x}_{0}\right.$, $\left.\mathbf{y}_{0}\right) \varepsilon C^{k}$, and the Jacobian matrix $[\partial \mathbf{F} / \partial \mathbf{x}]$ is nonsingular (regular) at $\left(\mathbf{x}_{0}, \mathbf{y}_{0}\right)$. Then there exists a neighbourhood of $\mathbf{y}_{0}$, say $N\left(\mathbf{y}_{0}\right)$, and a mapping $\mathbf{G}: N\left(\mathbf{y}_{0}\right) \rightarrow R^{n}$ such that $\mathbf{x}_{0}=\mathbf{G}\left(\mathbf{y}_{0}\right)$ and $\mathbf{G}\left(\mathbf{y}_{0}\right) \varepsilon C^{k}$ and $\mathbf{F}[\mathbf{G}((\mathbf{y}) \mathbf{y})]=0$ on $N(\mathbf{y})$. Moreover, we have 


$$
[\partial \mathbf{G} / \partial \mathbf{y}]^{t}\left|\mathbf{y}_{0}=-[\partial \mathbf{F} / \partial \mathbf{x}]^{-t}\right| \mathbf{x}_{0}[\partial \mathbf{F} / \partial \mathbf{y}]^{t} \mid \mathbf{x}_{0}, \mathbf{y}_{0} .
$$

The derivatives of the few lowest natural frequencies with respect to the structural design parameters can be obtained based on the reduced set of eigenvalues and eigenvectors obtained from the dynamic reduction method (see Fetterman and Noor 1987). In other words, we do not need to solve for all eigenvalues and eigenvectors to obtain the derivatives. Finally, by the chain rule, we can obtain the gradient of the objective function with respect to the structural design parameters.

A candidate optimal design for a smooth problem must satisfy the Kurash-Kuhn-Tucker necessary conditions (see Haftka and Kamat 1985; Luenberger 1984). Theorem 3.4.4 implies smoothness of the problem for general values of the structural design parameters, where $k=r$ in the control history. Herein we assume that this property holds for all values of the structural design parameters. This assumption is compatible with our experience in numerical studies. We use mathematical programming to find candidate optimal designs.

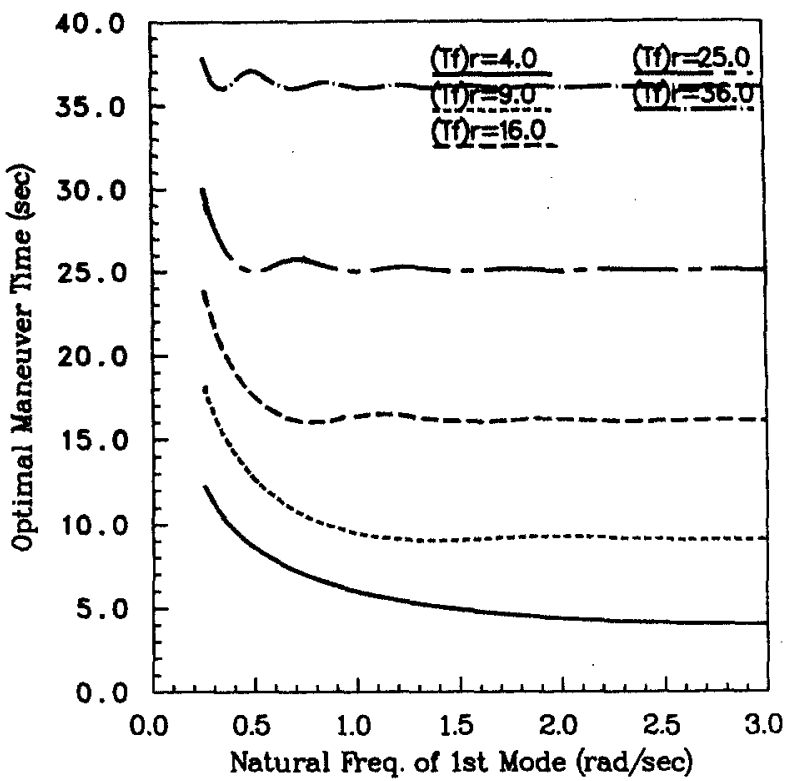

(TI) $r$ is the maneuver time of an equivalent rigid spacecraft

Fig. 2. Optimal maneuver time for the control design model with one flexible mode

From (14)-(15), $t_{f}^{*}$ is an implicit function of $\left(\omega, J^{*}\right)$ for the generic case where $k$ is equal to $r$. We show the behaviour of $t_{f}^{*}\left(\omega, J^{*}\right)$ for the simplest case where there is only one flexible mode in Figs. 2 and 3 . Let $\left(t_{f}\right)_{r}$ represent the maneuver time of an equivalent rigid spacecraft, thus $\left(t_{f}\right)_{r}=2 \sqrt{\theta J^{*} / U_{0}}$. In Fig. 2, for each curve $\left(t_{f}\right)_{r}$ is fixed and the natural frequency of the flexible mode varies from zero to a large value. In Fig. 3, for each curve the natural frequency of the flexible mode is fixed and $\left(t_{f}\right)_{r}$ varies from zero to a large value. We note that there exist several local optima along each curve in Fig. 2 and that each curve is smooth.

Furthermore we have obtained the following results. Assume a spacecraft has only one flexible mode.

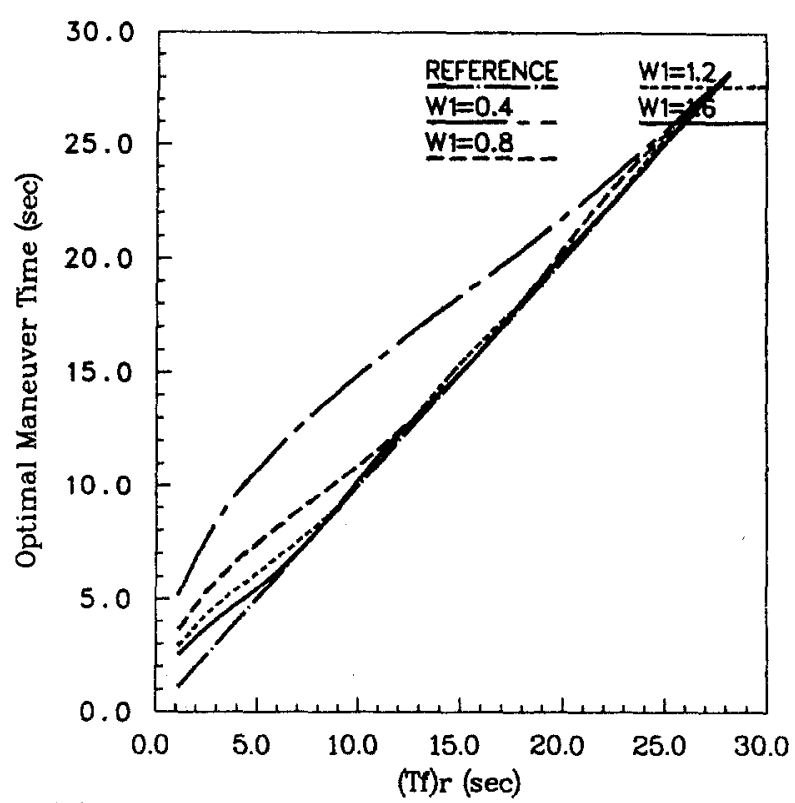

(Tf) $r$ is the maneuver time of an equivalent rigid spacecraft Reference is the curve: $\mathrm{Tf}=(\mathrm{TH}) \mathrm{r}$

Fig. 3. Optimal maneuver time for the control design model with one flexible mode

(i) For a spacecraft with very large $\omega_{1}$ (as shown in Fig. 2 , greater than $2.0 \mathrm{rad} / \mathrm{sec}$ ), which implies a very rigid spacecraft, $t_{f}^{*}$ is almost the same as $\left(t_{f}\right)_{r}$ for all the curves in Fig. 2.

(ii) For a very flexible spacecraft, with the natural frequency of the first flexible mode tending to be zero, the maneuver time always tends to increase substantially.

(iii) For a spacecraft with very large $\left(t_{f}\right)_{r}$ (as shown in Fig. 3 , greater than $24.0 \mathrm{sec}$ ), which implies the torquer limit is very small or the maneuver angle is very large, $t_{f}^{*}$ is almost the same as $\left(t_{f}\right)_{r}$ for all the values of $\omega_{1}$.

Of course, a typical spacecraft has more than one flexible mode, and we cannot say much about it. However, Figs. 2 and 3 provide important information. If the spacecraft is very flexible or the torquer limit is very large (usually this implies a very large maneuver speed), the result of the optimal design can provide substantial improvement.

\subsection{Problem solving algorithm}

The size of the control design model is chosen according to the analysis of the control spillover. In order to take advantage of Theorem 3.4.1, we assume that the control design model is fixed during the optimization and formulate the optimization procedure as follows.

$\mathcal{P}_{1}$ :

Begin with a reasonable baseline design of the spacecraft.

Step 0 . Set up the reduced model by (18) (set the value of $r$ ).

Step 1. Initialize the design variables. 
Step 2. Obtain the appendages for the current value of the design variables.

Step 3. Finite element analysis.

Step 4. Calculate the natural frequencies of the modes in the reduced model by the dynamic reduction method.

Step 5. Solve the time-optimal control problem to obtain the optimal maneuver time.

Step 6. Find the next values of the design variables by nonlinear programming, using the gradient computed by Theorem 3.4.5 and the chain rule.

Step 7. If the result is convergent, Step 8; otherwise go to Step 2.

Step 8. If the spillover constraint [ii of (2)] is satisfied, then stop; otherwise Step 0.

Although the algorithm $\mathcal{P}_{1}$ is able to solve the optimal structural design problem (2), unfortunately, in our experience, there exist a lot of numerical difficulties associated with it, i.e.

(i) to solve the time-optimal control problem, we need to know the number of switching times;

(ii) actually the set of nonlinear equations (14)-(16) admit many solutions, of which only one satisfies the inequality conditions (17). Thus, even though we have a good nonlinear equation solver, it would not be able to guarantee to find the solution we want;

(iii) the optimal design is likely to be such that the set of nonlinear equations tend to be singular;

(iv) solving nonlinear equations is time-consuming, and the solution is always approximate;

(v) many local solutions to the optimal design problem are likely to exist.

Considering all the above difficulties, it seems a formidable task to solve the optimal design problem by $\mathcal{P}_{1}$ without any simplification, especially if one expects to find the global optimal design. Therefore, we introduce approximate design methods as described in the next section.

\section{Approximate design methods}

The fundamental idea of this solution procedure is to formulate an approximate design problem without violating any constraint of the original problem. The solution of the approximate design problem is a 'near-optimal design' in the sense that there is little difference of objective function between the two solutions. We need to quantify the difference without solving the original problem and make it as small as possible. However, there is a trade-off between accuracy and effort for solving problems. Thus an important capability of the approximation algorithm is that we can adaptively upgrade the approximation procedure to obtain a reasonable result according to the specific application requirement. Since design models cannot exactly represent the real system, it is unreasonable to concern oneself so much about a relatively small improvement of accuracy of the solution based on a design model. In this section we introduce two approximate design methods: the adaptive frequency tuning method and the minorant method. The former is suitable for the single maneuver case; the latter requires more computation work, but is suitable for the multi-maneuver case.

\subsection{Frequency tuning approach}

Here we make two basic assumptions.

Assumption 4.1.1. The natural frequencies of the modes retained in the reduced order model can be freely assigned by adjusting the values of the design variables.

Assumption 4.1.2. During the design iteration, the mass distribution of the appendage is independent of the stiffness distribution, i.e. the total rotational moment of the spacecraft, $J^{*}$, does not change when the stiffness distribution is modified.

Assumption 4.1.1 is usually valid in that there is a sufficient number of structural design parameters and most of them have global influence on the spacecraft property. In general, we must have more design parameters than flexible modes retained in the control design model to obtain a global optimal design. Assumption 4.1.2 is usually appropriate for some applications such as when the appendages are made of I-beams, with truss structures, or the payload mass, compared with the structural mass, is dominant.

For the single maneuver case, consider (14) and (15), if for a spacecraft the natural frequencies of all modes in the reduced order model happen to satisfy

$\omega_{i} \bullet t_{f}^{*}=j_{i} \bullet 4 \pi, \quad i=1,2, \ldots, r$,

where $t_{f}$ is the maneuver time and $j_{i}$ is some integer multiplier. Then the solution in terms of switching times and the optimal maneuver time satisfies

$k=0$ and $t_{f}^{*}=2 \sqrt{\theta J^{*} / U_{0}}$.

It also satisfies the inequality condition (17). Thus we solve the time-optimal control problem for $\left\{\omega_{i}, i=1,2, \ldots, r\right\}$ satisfying (21). Moreover, (22) implies that there is no switch of the control history between 0 and $t_{f}^{*} / 2$, and only one switch at the mid-maneuver. This means that all flexible modes in the reduced model are dead beat at the end of the maneuver by the same control which maneuvers a rigid body of the same value of total rotational moment $J^{*}$. We now have the new optimization problem

$\mathcal{P}_{2} \quad \min _{\Xi} t_{f}^{*}=2 \sqrt{\theta J^{*} / U_{0}}$,

subject to the constraints I and II of (2) and (21).

Proposition 4.1.3. Under Assumptions 4.1.1 and 4.1.2 above, the solution of $\mathcal{P}_{2}$ solves the original problem (2), and is a global optimum.

Proof. Since $\theta$ and $U_{0}$ are fixed constants during the optimization, $\mathcal{P}_{2}$, Assumption 4.1.2 implies that the minimization of $t_{f}^{*}=2 \sqrt{\theta J^{*} / U_{0}}$ will not be influenced by the constraint (21). Thus the solution of $\mathcal{P}_{2}$ is the same as that of 
$\mathcal{P}_{1}$, the original problem. Assumption 4.1.1 further implies that we can obtain the solutions of $\mathcal{P}_{2}$ as $2 \sqrt{\theta L^{*} / U_{0}}$, where $J^{*}$ is the global minimum of the rotation moment of all feasible designs [subject to the constraints (2)]. Therefore this is the result of the global optimum.

The rigid-body control strategy is the simplest to implement and we do not need to solve any nonlinear equations (14)-(16). Furthermore, the optimal design of the appendage which satisfies (21) may be very flexible (in the sense that natural frequencies of the first few flexible modes are very small) and very light (in the sense that $J^{*}$ is small). This idea for design appears to be original.

\subsection{Adaptive upgrade algorithm}

Unfortunately, Assumption 4.1.2 is not always satisfied in general applications. For example, in designing an appendage of rectangular cross-section with high density material, the stiffness is highly coupled with the design of the mass distribution. Actually, $\mathcal{P}_{2}$ implicitly assumes that the global optimal design of the appendages is such that the time-optimal control is the same as the rigid-body control strategy. We restrict ourselves to solving the original problem in a subspace of the feasible design variable space. Therefore, the result of $\mathcal{P}_{2}$, in general, does not apply and needs to be modified or upgraded.

We first quantify the index of approximation, associated with an approximation design problem, as the difference of objective function between the exact optimal design (the solution of the original problem) and the solution of the approximate design problem. Let $t_{f}$ be the maneuver time of the exact optimal design, which is equal to the minimum of $t_{f}^{*}$ over the entire feasible design space. Also we note that $\mu$ is equal to $\int p\left(\theta_{i}\right) \bullet t f\left(\theta_{i}\right) \mathrm{d} \theta_{i}$.

Let $t f^{a}, \mu^{a}$ indicate the approximated solution of $t f$ and $\mu$, respectively. Then we introduce the following:

index of approximation : $\mathcal{E}_{0}=\left|t f^{a}-t f\right|$ or $\left|\mu^{a}-\mu\right|$.

An approximate solution is better if its index of approximation is smaller. However, this does not mean that the approximate and exact designs are close to each other. For example, they may be substantially different in shape. In order to avoid difficulties in computing $t f$, we modify (24)

$\mathcal{E}_{1}=\left|t f^{a}-\mathcal{L}^{b}(t f)\right|$ or $\left|\mu^{a}-\mathcal{L}^{b}(\mu)\right|$

where $\mathcal{L}^{b}(\bullet)$ is a lower bound of $\bullet$ and is very easy to compute. Also we clearly have

$\mathcal{L}^{b}(\mu)=\int p\left(\theta_{i}\right) \mathcal{L}^{b}\left[t f\left(\theta_{i}\right)\right] \mathrm{d} \theta_{i}$

There are two ways to define such a lower bound.

1. The maneuver time for a rigid spacecraft with the least feasible total rotational moment $\underline{J}^{*}$

$$
\mathcal{L}_{1}^{b}(t f)=2 \sqrt{\theta \underline{J}^{*} / U_{0}}
$$

It is usually unreasonable to define the lower bound in this way because (27) is very conservative. The appendage with the least total rotational moment is usually too slender, too flexible and is likely to require a long maneuver time.

2. The optimal maneuver time of the optimal design which is based on a reduced model with only one flexible mode. Let the superscript 1 of $t f$ indicate that the value is based on a reduced model with only one flexible mode. Thus,

$\mathcal{L}_{2}^{b}(t f)=t f^{1}=$ minimum of $t_{f}^{* 1} \quad$ over the entire

feasible design space.

Since we need more maneuver time for a control design model with more than one flexible mode, we know $t f^{1}$ is a lower bound of the maneuver time for the design problem of any reduced order model. We need some computation effort to calculate $t f^{1}$; however, the calculation is not very difficult. It is more reasonable to define the lower bound of the maneuver time to be $t f^{1}$.

We propose the modified approximate problem $\mathcal{P}_{3}$ according to the following facts.

Fact 4.2.1. For a specified reduced order model with $r$ flexible modes, the whole feasible design space consists of the following sets:

$\mathcal{D}_{0}:\{\xi:$ the time-optimal control history of this design has only switch at mid-maneuver, without any switch between 0 and $t_{f}^{*} / 2$;

$\mathcal{D}_{1}:\{\xi$ : the time-optimal control history of this design has one switch at mid-maneuver and at most one switch between 0 and $t_{f}^{*} / 2$;

$\mathcal{D}_{2}:\{\xi:$ the time-optimal control history of this design has one switch at mid-maneuver and at most two switches between 0 and $\left.t_{f}^{*} / 2\right\}$;

and we have set the relationship

$\mathcal{D}_{0} \subseteq \mathcal{D}_{1} \subseteq \mathcal{D}_{2} \subseteq \mathcal{D}_{3} \subseteq \ldots$

Fact 4.2.2. From Fact 4.2.1, we know $\min \left\{\mu^{*}(\xi)\right.$ or $t_{f}^{*}(\xi)$ of problem (2)\} over the subspace $\mathcal{D}_{i} \geq \min \left\{\mu^{*}(\xi)\right.$ of problem (2)\} over the subspace $\mathcal{D}_{i+1}$.

Fact 4.2.3. The exact solution of problem (2) is $\min \left\{\mu^{*}(\xi)\right.$ or $t_{f}^{*}(\xi)$ of problem (2)\} over the subspace $\mathcal{D}_{r}$ for some $r \geq 0$. (31)

Actually, the solution of $\mathcal{P}_{2}$ is nothing but $\min \left\{t_{f}^{*}(\xi)\right.$ of problem (2)\} over the subspace $\mathcal{D}_{0}$. Similarly, $\mathcal{P}_{3}$ is the problem of solving for $\min \left\{t_{f}^{*}(\xi)\right.$ of problem (2)\} over the subspace $\mathcal{D}_{i}, i \geq 0$, with adaptively upgrading by increasing $i$, and with a stopping criterion based on sufficiently small change of improvement of the index of approximation (24). We eventually obtain the exact optimal design if the upgrade 
continues. However, we have restricted ourselves to solving for $i \leq 2$, to avoid difficulty in computation.

$\mathcal{P}_{3}$ :

Step 1. Let $k=0$, and solve $\mathcal{P}_{2}$.

Step 2. If the solution is not acceptable, Step 3; otherwise stop.

Step 3. Let $k=k+1$.

Step 4. Solve $\min _{\Xi} t_{f}^{*}$ subject to the constraints I and II of (2), and the number of switching times is $k$ [in other words, for the specified value of $k$ in (14)-(17), we obtain a solution].

Step 5. Obtain the index of approximation $\mathcal{E}$. If there is no relative improvement of it, stop; otherwise Step 3.

However, it is difficult to obtain the subset $\mathcal{D}_{i}$, for some $i \geq 0$, of the whole feasible design space. To solve the problem efficiently, we need explicit numerical expressions to obtain $\mathcal{D}_{i}$. From Corollary 3.2.5, we have the following proposition. Proposition 4.2.4. Step 4 of $\mathcal{P}_{3}$ can be replaced with the following problem:

$\mathcal{P}_{3}^{*} \quad \min _{\Xi} t_{f}^{*} k$

subject to the constraints I and II of (2), and

$\cos \left(\omega_{i} t_{f}^{*} / 2\right)-2 \cos \left(\omega_{i} t_{k}\right)+2 \cos \left(\omega_{i} t_{k-1}\right)-\ldots+$

$+2(-1)^{k} \cos \left(\omega_{i} t_{1}\right)+(\stackrel{-}{-1})^{k+1}=0$,

$i=k+1, k+2, \ldots, r$,

where the superscript $k$ indicates that there are $k$ flexible modes in the control design model.

Proof. Since there are generally $k$ switching times for $t_{f}^{*} k$, it is evident that $\mathcal{P}_{3}^{*}$ is from the results of Corollary 3.2.5.

The most important advantage of $\mathcal{P}_{3}$ is that we only need to solve a small set of nonlinear equations. The difficulty to solve nonlinear equations increases very dramatically as the number of nonlinear equations increases. Another advantage is that we tend to avoid the singular cases, where the Jacobian of (14) and (15) is singular, such as where two switching times tend to each other or one tends to zero, because we solve for the optimal design from a subset $\mathcal{D}_{i}$, where $i$ begins from the lowest possible number of switching times.

\subsection{The minorant design problem}

$\mathcal{P}_{2}$ and $\mathcal{P}_{3}$ are not suitable for the general multiple maneuver case because it is difficult to find $\omega_{i}, i=1,2, \ldots, r$ which satisfy (21) for many different maneuvers, $\left\{\theta_{i}\right\}$. In this section we discuss an algorithm, the minorant method, which is more difficult to implement, but is suitable for the multiple maneuver case. While solving the time optimal control problem, we find that for any design of spacecraft, $t_{f}^{* r+1} \geq t_{f}^{* r}$; however, the difference becomes smaller as $r$ increases. From our numerical studies, it is observed that the maneverability is most influenced by the total rotational moment $J^{*}$, and then by the first few flexible modes. An appendage with smaller total rotational moment or with more rigidity, in the sense that the natural frequencies of the lowest few flexible modes are large, tends to be very maneuverable.

$\mathcal{P}_{4}$ is based on the following assumption and fact.

Assumption 4.3.1. For any feasible design of the spacecraft $\xi \varepsilon \Xi$, we have $\left|t_{f}^{*}(\xi)^{i+2}-t_{f}^{*}(\xi)^{i+1}\right| \leq\left|t_{f}^{*}(\xi)^{i+1}-t_{f}^{*}(\xi)^{i}\right|, i \geq$ 0 , where the superscript $i$ indicates that the quantity is obtained based on a reduced model with $i$ flexible modes.

Fact 4.3.2. $\left|t f^{i+2}-t f^{i+1}\right| \leq\left|t f^{i+1}-t f^{i}\right|, i \geq 0$, and $\left|t f^{i}-t f^{r}\right| \rightarrow 0$ as $r$ and $i$ are sufficiently large. Furthermore, $\left|\mu^{i+2}-\mu^{i+1}\right| \leq\left|\mu^{i+1}-\mu^{i}\right|, i \geq 0$, and $\left|\mu^{i}-\mu^{r}\right| \rightarrow 0$ as $r$ and $i$ are sufficiently large.

$\mathcal{P}_{4}:$

Step 1. Let $i=0$, and solve $\mu^{i}$ by $\mathcal{P}_{1}$.

Step 2. Obtain the index of improvement $\mathcal{E}$. If there is only a small improvement, stop; otherwise $i=$ $i+1$. Go to Step 1 .

The exact optimal design can be obtained for $i=r$. However, we do not go beyond $i \geq 2$. The capability of $\mathcal{P}_{4}$ will be investigated later with numerical examples.

\section{Numerical examples}

In our example, we design appendages by adjusting their cross-section. We use practical examples with realistic scale and material. Furthermore, we try to investigate the design of large flexible space structures, such as large antenna or space stations.

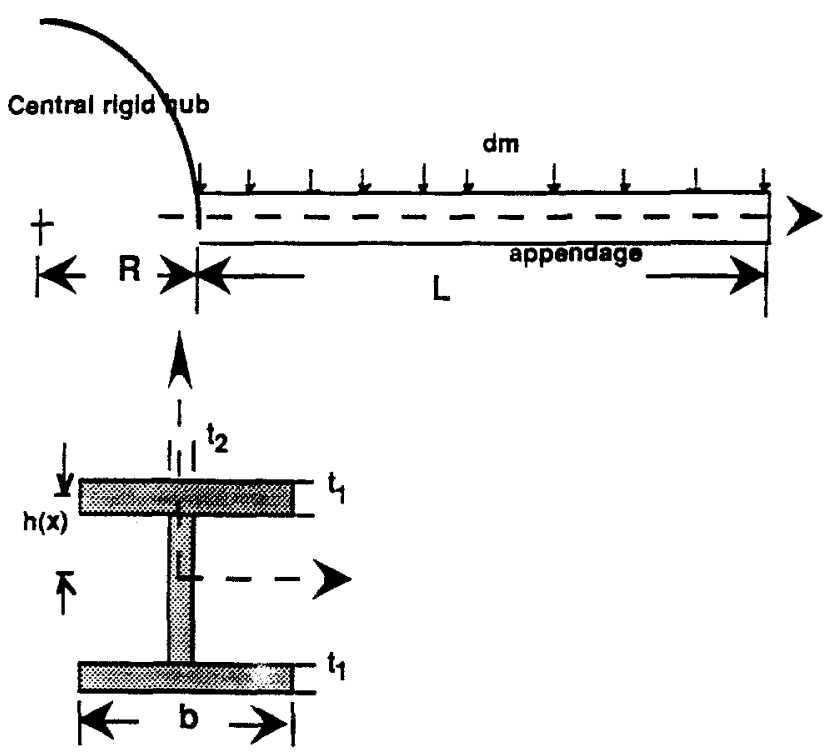

I-Beam Crosesection

Fig. 4. Design of the cross-section of appendages

In the following, we perform the modal analysis with the finite element method and model the flexible spacecraft with 
one rigid body mode and 60 flexible modes (each appendage is discretized into 30 uniform beam elements). There are $r$ flexible modes, obtained by the dynamic reduction method, retained in the reduced order model for control design. The reduced order model is specified according to the postmaneuver spillover constraint. In the examples, we specify the maximum angular deviation of the central rigid body postmaneuver as $0.05 \mathrm{deg}$. The appendages are $I$-beams (as shown in Fig. 4). Our goal is to obtain the optimal flange depth distribution of the appendages, and we assume the width of the web, and thickness of the web and flange to be constant. The flange depth is symmetric about a central line passing through the cross-section. We use two spline polynomials as the assumed shape functions to describe the half flange depth

$h_{1}(x)=c_{1}+c_{2}(x / L)+c_{3}(x / L)^{2}+c_{4}(x / L)^{3}$,

for $0 \leq x \leq L / 2$,

$h_{2}(x)=h_{1}(L / 2)+h_{1}^{\prime}(L / 2)(x-L / 2)+c_{5}(x-L / 2)^{2} / L^{2}+$

$+c_{6}(x-L / 2)^{3} / L^{3}$ for $L / 2 \leq x \leq L$,

where $c_{i}, i=1,2, \ldots, 6$ are design variables.

All design variables $c_{i}, i=1,2, \ldots, 6$ are scaled to the same order of magnitude (independent of $L$ ) in order to obtain proper search directions in nonlinear programming. For practical reasons it is reasonable that $h(x)$ and $\mathrm{d} h(x) / \mathrm{d} x$ be continuous at $x=L / 2$. Each domain of the polynomial is discretized into 15 elements in the finite element analysis.

We consider a spacecraft with two identical flexible appendages. The constraints considered in this problem are the resource constraint, minimal and maximal depth of the flange, and the postmaneuver control spillover constraint. For simplicity, we assume the appendages are made of a single uniform material.

We begin solving the problem by finding a reasonable number of modes in the reduced order model. We use a reasonable baseline design with uniform flange depth equal to $4.00 \mathrm{~cm}$. As shown in Table 1, we note that it is appropriate to retain three flexible modes for a postmaneuver maximum angular deviation to be guaranteed less than $0.05 \mathrm{deg}$.

\section{Spacecraft data}

appendage material density, $\rho$ appendage material elasticity, $E$ radius of the rigid central body, $R$ mass of the rigid central body length of one appendage, $L$ maximum torque available, $U_{0}$ width of the web, $b$ thickness of the web, $t 1$ thickness of the flange, $t 2$ distributed pay load mass, $d m$ concentrated pay load mass (at $x=L$ ), $M$

$1880.00 \mathrm{~kg} / \mathrm{m}^{3}$ $2.76 \mathrm{E} 11 \mathrm{~N} / \mathrm{m}^{2}$ $12.00 \mathrm{~m}$ $4500.00 \mathrm{~kg}$ $50.00 \mathrm{~m}$ $3.0 \mathrm{E} 4 \mathrm{~N}-\mathrm{m}^{*}$ $5.00 \mathrm{~cm}$ $1.75 \mathrm{~cm}$ $0.75 \mathrm{~cm}$ $9.00 \mathrm{~kg} / \mathrm{m}$ none

\section{Design constraints}

the resource constraint of two appendages the minimal flange depth
$450.0 \mathrm{~kg}$ $2.00 \mathrm{~cm}$

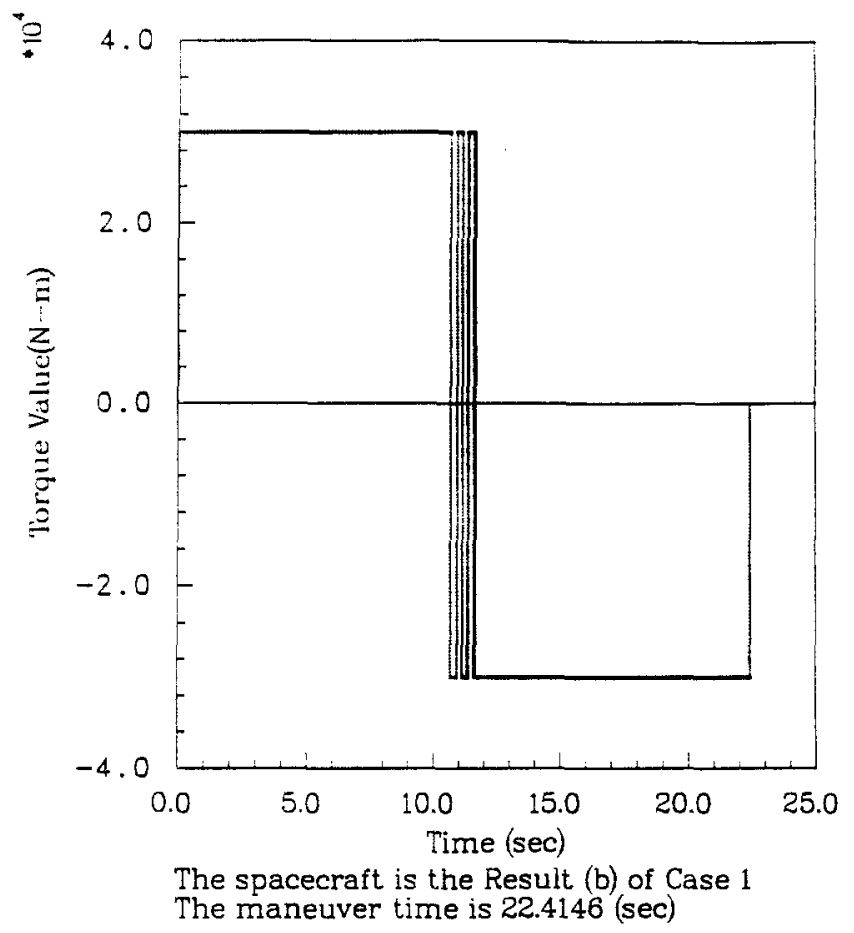

Fig. 5. Time optimal control history for the design model with three flexible modes

the maximal flange depth

$12.00 \mathrm{~cm}$

Case 5.1. Single maneuver case

Command slew angle, $\theta$

$90.00 \mathrm{deg}$.

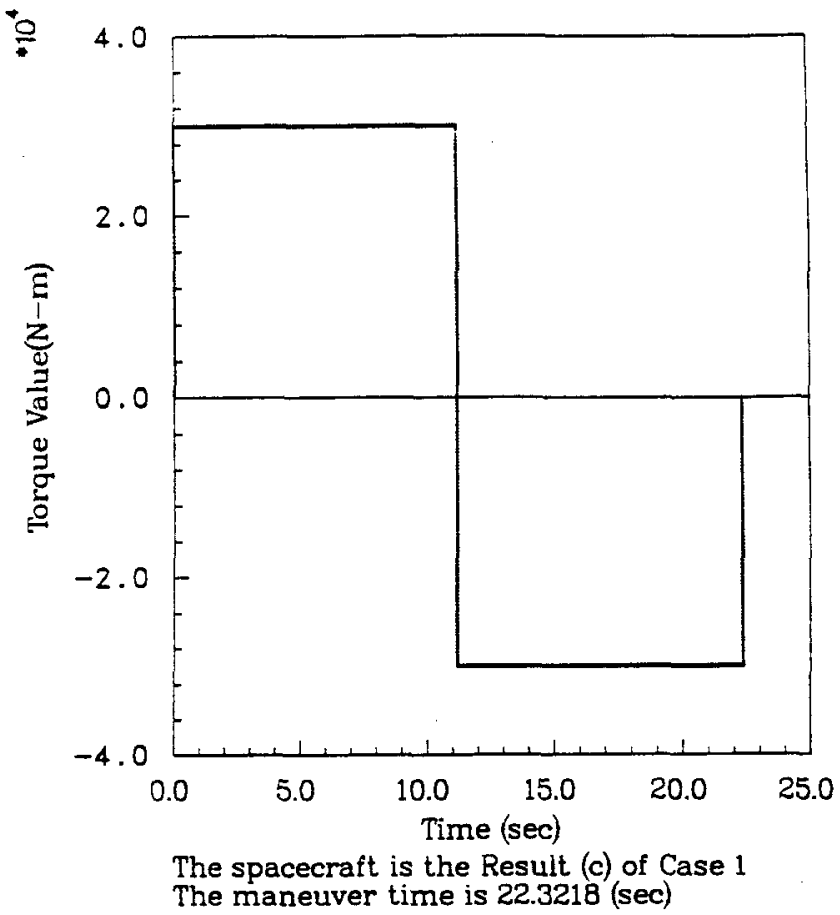

Fig. 6. Time optimal control history for the design model with three flexible modes

* $U_{0}$ represents the torquer on the rigid central body 


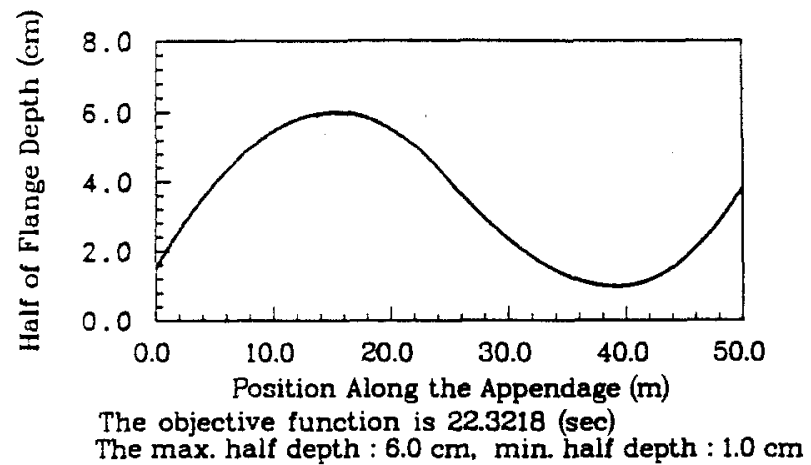

Fig. 7. Optimal design of the spacecraft for Case 5.1. Flange depth distribution of the flexible appendages

Thus the exact solution is $t f$, which is equal to $t_{f}^{* 3}$ over the entire feasible design space.

Results

$\mathcal{L}_{1}^{b}(t f)=2 \sqrt{\theta J^{*} / U_{0}}=21.9814 \mathrm{sec}$, but $t_{f}^{* 3}$ of this design is $24.6213 \mathrm{sec}$.

$\mathcal{L}_{2}^{b}(t f)=t f^{1}=22.3126 \mathrm{sec}$ and $t_{f}^{* 3}$ of this design is $22.41457 \mathrm{sec}$. The switching times between 0 and $t_{f}^{* 3}$ of the time-optimal control history are $1.5547 \mathrm{E}-8,0.21945$, $0.48124 \mathrm{sec}$ (one switching time is almost zero). The control time history is shown in Fig. 5 .

From $\mathcal{P}_{2}: t f$ over the domain $\mathcal{D}_{0}$ is $22.3218 \mathrm{sec}$. The control time history is shown in Fig. 6. Let it be $t f^{a}$, then $\left|t f^{a}-\mathcal{L}_{2}^{b}(t f)\right|=9.2 \mathrm{E}-3$. We can accept this design as the solution (as shown in Fig. 7). The time histories of the attitude and attitude rate of the rigid central body for the spacecraft are shown in Figs. 8 and 9.

The properties of this optimal design of Case 5.1 are as follows.

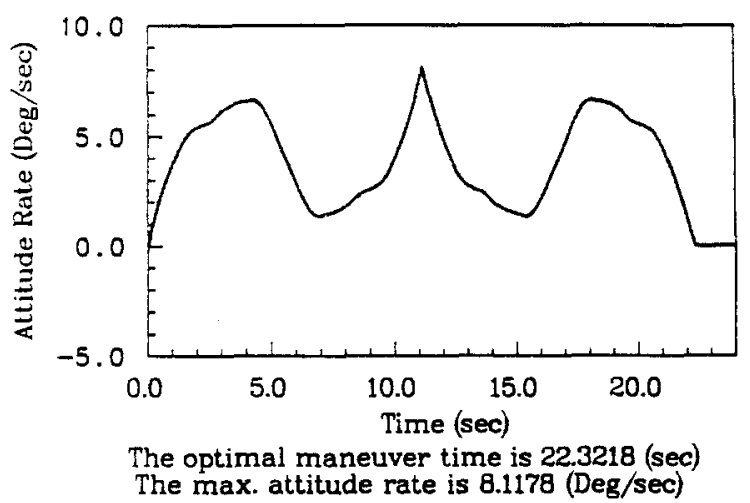

Fig. 8. Time history of the rigid central body attitude rate for the optimal design of Case 5.1

Structural mass of two appendages

Total pay load mass along the appendages

$379.687 \mathrm{~kg}$. $900.00 \mathrm{~kg}$.

Total mass of the spacecraft $5779.687 \mathrm{~kg}$.

(For the appendage: payload mass/structural mass = $237.02 \%$, and for the spacecraft: appendage mass/spacecraft mass $=22.14 \%$ ).

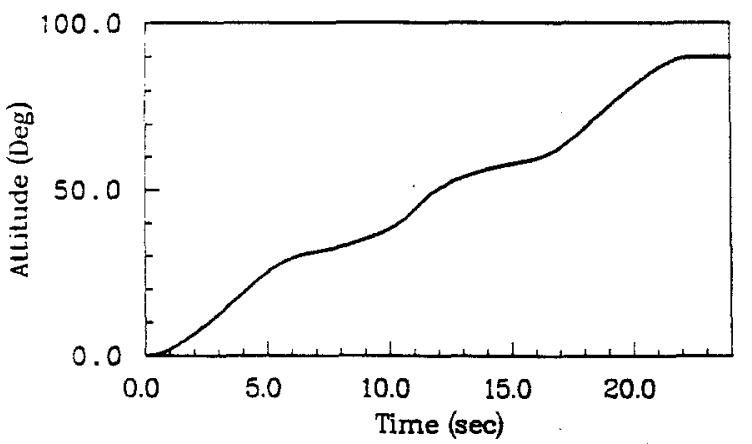

The optimal maneuver time is 22.3218 (sec)

The maneuver angle of the rigid central body is 90 (Deg)

The max. post-maneuver attitude error is less than 0.05 (Deg)

Fig. 9. Time history of the rigid central body attitude for the optimal design of Case 5.1

Total rotational moment

$2375330.68 \mathrm{~kg}-\mathrm{m}^{2}$.

Natural frequency $\omega_{i}, i=1,2, \ldots, 4,0.5642,1.6942$, $4.4738,8.9745(\mathrm{rad} / \mathrm{sec})$.

The maximum postmaneuver angle deviation of the central rigid body due to the uncontrolled modes is $0.00908 \mathrm{deg}$. Number of switches between 0 and $t_{f}^{*} / 2$ of the time-optimal control history: none.

Case 5.2. General multiple maneuvers. The set of maneuvers is $\left\{\theta_{i}\right\}=\{9,15,30,45,60,90(\mathrm{deg})\}$, and it is assumed that they occur at the same frequency. Thus the objective function (maneuverability index) is

$\mu(\xi)=\frac{1}{6} \sum_{i=1}^{6} t_{f}^{*}\left(\theta_{i}\right)$

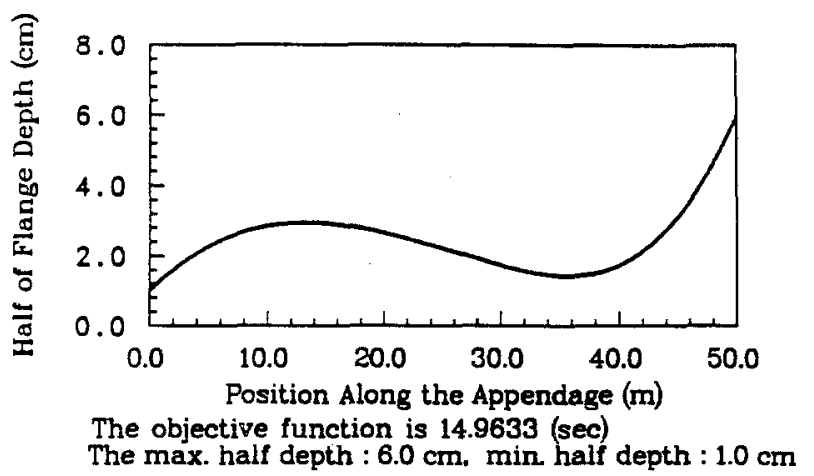

Fig. 10. Optimal design of the spacecraft for Case 5.2. Flange depth distribution of the flexible appendages

The solution $\mu$ equals $\mu^{* 3}$ over the entire feasible design space.

Results

$$
\mathcal{L}_{1}^{b}(\mu)=2 \sqrt{\theta J^{*} / U_{0}}=13.1753 \mathrm{sec} .
$$

- $\mathcal{L}_{2}^{b}(\mu)=\mu^{1}=15.0436 \mathrm{sec}$, and $\mu^{* 3}$ for this design is 15.30617 sec. As $\mathcal{P}_{4}$ : if we let $\mu^{a}=15.30617$, we have $\left|\mu^{a}-\mathcal{L}_{2}^{b}(\mu)\right|=0.26257$, as $1.7454 \%$. 
- $\mu^{2}$ is $14.8580 \mathrm{sec}$, and $\mu^{* 3}$ for this design is 14.96326 sec. As $\mathcal{P}_{4}$ : if we let $\mu^{a}=14.96326$, we have $\left|\mu^{a}-\mathcal{L}_{2}^{b}(\mu)\right|=$ 0.06526 , as $0.4392 \%$. We accept it as the solution (as shown in Fig. 10).

- We investigate the exact solution by $\mathcal{P}_{1}$ and obtain $\mu^{3}$ is $14.9455 \mathrm{sec}$.

The properties of this optimal design of Case 2 are as follows.

Stuctural mass of two appendages

$425.075 \mathrm{~kg}$.

Total pay load mass along the appendages

$900.00 \mathrm{~kg}$.

Total mass of the spacecraft

$5825.075 \mathrm{~kg}$.

(For the appendage: payload mass/structural mass = $211.72 \%$, and for the spacecraft: appendage mass/spacecraft mass $=22.75 \%$ ).

Total rotational moment

$2379168.55 \mathrm{~kg}-\mathrm{m}^{2}$.

Natural frequency $\omega_{i}, i=1,2, \ldots, 4,0.8460,2.0276,5.5051$, $10.6193(\mathrm{rad} / \mathrm{sec})$.

The maximum postmaneuver angle deviation of the central rigid body due to the uncontrolled modes is $0.02436 \mathrm{deg}$.

Number of switches between 0 and $t_{f}^{*} / 2$ of the time optimal control history: three.

The postmaneuver control spillover of both cases are less than the specified tolerance, $0.05 \mathrm{deg}$. The results of this example show that we can find an optimal design for a structure which is very flexible (the first natural frequency of the spacecraft of Case 5.1 is $0.5642 \mathrm{rad} / \mathrm{sec}$ and that of Case 5.2 is $0.8460 \mathrm{rad} / \mathrm{sec})$. The material resource constraint is not important (inactive in both cases). The approximate methods can efficiently find the solution and avoid computational difficulties. The indices of approximation of both cases are well acceptable.

\section{Conclusions and future work}

The combined design of structure and control for optimal maneuverability with application to flexible spacecraft has been considered. The spacecraft is modelled as a linear, elastic, undamped, nongyroscopic system. We have developed a theoretical and practical framework for solving this problem. The main features of the present work are as follows.

(i) The problem formulation is consistent with bang-bang forms of time-optimal controls.

(ii) The performance degradation constraint is considered in the design problem.

(iii) The optimal design problem has been shown to be well defined. There always exists a solution.

(iv) The structural optimization is done by finite element analysis and mathematical programming.

(v) The gradient of the objective function is computed using the implicit function theorem.

(vi) Efficient and practical approximate methods have been developed.
The use of time optimal control of bang-bang type for flexible spacecraft maneuvers has been criticized, e.g. by Thompson et al. (1990): "near bang-bang controls offer the shortest maneuver time; however, control spillover into the flexible modes will introduce structural vibrations, perhaps to an unacceptable degree". However, as the result of our examples suggest, with the present optimal structural design formulation we obtain a design which satisfies the control spillover constraint even while the system is subject to a bang-bang type optimal control. Furthermore, the maneuver time is very close to its lower bound. Also, we have found that if the spacecraft is very large and flexible, the maneuver time is substantially larger than that of an equivalent rigid spacecraft. For example, as shown in result (a) of Case 5.1, the optimal maneuver time for the flexible spacecraft is larger than the equivalent rigid spacecraft by an amount over $12 \%$. Thus the idea that the "the minimum time solution for a rigid spacecraft can serve as an initial approximation to the nearminimum time solution for a flexible structure" (Thompson et al. 1990), might not be applicable in some cases. The combined optimal design formulation for spacecraft of this kind may provide significant benefit.

The problem solving procedure developed herein can be applied to the general design of spacecraft possessing appendages made of longeron (truss structures) or composite material. The finite element analysis can be efficiently used for modelling. Our experience with various numerical examples leads to the following general conclusions.

(i) The best structural designs are often those for which the designs of mass distribution and stiffness distribution have very little coupling.

(ii) The benefit of multiple controls is not apparent since we can use scalar control to achieve good results.

The spacecraft structure has been modelled to be linear, with small displacement and inextensible deformation. However, as the results of numerical examples show, we may obtain an optimal design which is very flexible. This performance for a realistic system which violates these assumptions is worth investigating. The constraint of structural dynamic response, such as maximal displacement and stress, should also be considered in the example. Those topics are indicated for future study.

\section{References}

Athanas, M.; Falb, P. 1966: Optimal control. New York: MacGraw-Hill

Barbieri, E.; Ozguner, U. 1988: Rest-to-rest slewing of flexible structures in minimum time. Proc. IEEE Conf. Decision and Control (held in Austin, TX), pp. 1633-1638

Ben-Asher, J.; Burns, J.A.; Cliff, E.M. 1987: Time optimal slewing of flexible spacecraft. Proc. IEEE Conf. Decision and Control (held in Los Angeles, CA), pp. 524-528

Bendsøe, M.P; Olhoff, N.; Taylor, J.E. 1987: On the design of structure and controls for optimal performance of actively controlled flexible structures. Mech. Struct.\& Mach. 51, 265-295 
Bodden, D.S.; Junkins, J.L. 1985: Eigenvalue optimization algorithms for structure/controller design iterations. J. Guidance, Control and Dynamics 8, 697-706

Breakwell, J.A. 1981: Optimal feedback slewing of flexible spacecraft. J. Guidance, Control and Dynamics 4, 472-479

Doyle, J.C. 1987: Guaranteed margins for LQG regulators. IEEE Trans. Auto. Control AC-23, 756-757

Doyle, J.C.; Stein, G. 1979: Robustness with observer. IEEE Trans. Auto. Control AC-24, 607-611

Fetterman, T.L.; Noor, A.K. 1987: Computational procedures for evaluating the sensitivity derivatives of vibration frequencies and eigenmodes of framed structures. NASA Contractor Report 4099

Fujii, H.; Ishijima, S. 1989: Mission-function control for slew maneuver of a flexible space structure. J. Guidance, Control and Dynamics 12, 858-865

Garcia, C.B.; Zangwill, W.I. 1981: Pathways to solutions, fixed points and equilibria. New Jersey: Prentice-Hall

Haftka, R.T.; Kamat, M.P. 1985: Elements of optimal structural design. Dordrecht: Kluwer

Haftka, R.T.; Martinovic Z.N.; Hallauer, W.L. Jr. 1985: Enhanced vibration controllability by minor structural modification. AIAA J. 23, 1260-1266

Hale, A.L.; Lisowski, R.J. 1985: Characteristic elastic systems of time-limited optimal maneuvers. J. Guidance, Control and $D y$ namics 8, 628-636

Hale, A.L.; Lisowski, R.J.: Optimal simultaneous structural and control design of maneuvering flexible spacecraft. Proc. 4th VPI \& $S U / A I A A$

Hale, A.L.; Lisowski, R.J.; Dahl, W.E. 1984: Optimal simultaneous structural and control design of maneuvering flexible spacecraft. J. Guidance, Control and Dynamics 8, 86-93

Hartman, P. 1982: Ordinary differential equations. Boston: Birkhauser

Khot, N.S. 1988: An integrated approach to the minimum weight and optimum control design of space structures. In: Large space structures: dynamics and control, pp. 355-363. Berlin, Heidelberg, New York: Springer

Khot, N.S.; Eastep, F.E.; Venkayya, V.B. 1985a: Optimal structural modifications to enhance the optimal active vibration control of large flexible structures. AIAA Paper, 85-0627

Khot, N.S.; Eastep, F.E.; Venkayya, V.B. 1985b: Simultaneous optimal structural/control modifications to enhance the vibration control of large of a large flexible structure. AIAA Paper, 85-1925

Khot, N.S.; Oz, H.; Grandhi, R.V.; Eastep, F.E.; Venkayya, V.B. 1988: Optimal structural design with control gain norm constraint. AIAA J. 26, 604-611

Lehtomaki, N.A.; Sandell, N.R.; Athanas, M. 1981: Robustness results in linear quadratic Gaussian based multivariable control design. IEEE Trans. Auto. Control AC-26, 75-93

Lim, K.B.; Junkins, J.L. 1989: Robustness optimization of structural and controller parameters. J. Guidance, Control and $D y$ namics 12, 89-96

Luenberger, D.G. 1984: Linear and nonlinear programming. New York: Addison-Wesley

Lust, R.V.; Schmit, L.A. 1988: Control-augmented structural synthesis. AIAA J. 26, 86-95
Meirovitch, L. 1967: Analytical methods in vibrations. New York: MacMillan

Onoda, J.; Haftka, R.T. 1987: An aproach to structural/control simultaneous optimization for large flexible spacecraft. AIAA J. 25, 1133-1138

Safanov, M.G.; Athanas, M. 1977: Gain and phase margin for multiloop LQG regulators. IEEE Trans. Auto. Control AC-22, 173-179

Singh, G.; Kabamba, P.T.; McClamroch, N.H. 1989: Planar, timeoptimal, rest-to-rest slewing maneuvers of flexible spacecraft. $J$. Guidance, Control and Dynamics 12, 71-81

Thompson, R.C.; Junkins, J.L.; Vadali S.R. 1989: Near-minimum time open-loop slewing of flexible vehicles. J. Guidance, Control and Dynamics 12, 82-88

Thompson, R.C.; Junkins, J.L.; Vadali S.R. 1990: Near-minimum time close-loop slewing of flexible spacecraft. J. Guidance, Control and Dynamics 13, 57-65

Turner, J.D.; Chun, H.M. 1984: Optimal distributed control of flexible spacecraft during a large-angle maneuver. J. Guidance, Control and Dynamics 7, 257-264

Vadali, S.R. 1986: Variable-structure control of spacecraft largeangle maneuvers. J. Guidance, Control and Dynamics 9, 235-239

Van der Velde, W.E.; He, J. 1983: Design of space structure control systems using on-off thrusters. J. Guidance, Control and $D y$ namcis 6, 53-60

\section{Appendix}

Corollary $A 1$. Let there be $k$ switching times between 0 and $t_{f}^{*} / 2$ of the optimal control history for control design model with $r$ flexible modes. Let the switching times be $t_{j}, j=1,2, \ldots, k$, and the optimal maneuver time be $t_{f}^{*}$. Suppose that $k=r$. Then the optimal maneuver time and the switching times satisfy as necessary and sufficient conditions

(i) (14) and (15) admit a solution for $\left\{t_{j}, j=1,2, \ldots, k\right.$ and $\left.t_{f}^{*}\right\}$ which is regular;

(ii) $\left\{t_{j}, j=1,2, \ldots, k\right.$ and $\left.t_{f}^{*}\right\}$ satisfy (16) and inequalities (17).

Proof. The sufficiency is shown in a paper by Singh et al. (1989).

Necessity: condition (ii) is evident. We only show condition (i). Since there are $k+1$ unknowns, $\left\{t_{j}, j=1,2, \ldots, k\right.$ and $\left.t_{f}^{*}\right\}$, and $k+1$ equations $\{(14),(15)\},\{(14),(15)\}$ should admit a solution for $\left\{t_{j}, j=1,2, \ldots, k\right.$ and $\left.t_{f}^{*}\right\}$. Furthermore, the coefficient matrix in (16) is exactly the transpose of the Jacobian of $\{(14),(15)\}$. If for the solution $\{(14)$, (15) $\}$ is not regular, which implies that the coefficient matrix in (16) is singular, this implies that there exists an infinite number of solutions for $\left\{p_{0}^{i}, i=1,2, \ldots, r\right\}$. This contradicts the uniqueness of the solution for the costates of the time optimal control problem. Thus $\{(14),(15)\}$ is regular.

Theorem A1. For our time-optimal control problem, the solution of the costate vector is unique. 
Proof. The proof primarily follows the heuristic proof of the minimum principle (see Athanas and Falb 1966, Sections 516 , pp. 308-340), which makes use of geometric ideas.

Consider the time optimal control problem

$\min J=t_{f}(\theta)$

subject to

$\dot{\mathbf{x}}=\mathbf{A x}(\mathbf{t})+\mathbf{B u}(\mathbf{t}), \quad\left|u_{j}(t)\right| \leq U_{j} ; j=0,1, \ldots, m$,

$\mathbf{x}(0)=(0,0, \ldots, 0)^{t}, \quad \mathbf{x}\left(t_{f}\right)=(\theta, 0, \ldots, 0)^{t}$.

Introduce an auxilary variable $x_{0}=t$, which is the cost of this problem. Thus

$\dot{x}_{0}=1$ and $x_{0}(0)=0$. We have $J=x_{0}\left(t_{f}\right)$.

Let $\mathbf{y}=\left[\begin{array}{ll}x_{0} & \mathbf{x}^{t}\end{array}\right]^{t}, \overline{\mathbf{u}}=\left[\begin{array}{ll}1 & \mathbf{u}^{t}\end{array}\right]^{t}$

$\overline{\mathbf{A}}=\left|\begin{array}{ll}0 & 0 \\ 0 & \mathbf{A}\end{array}\right|$ and $\overline{\mathbf{B}}=\left|\begin{array}{cc}1 & \mathbf{0} \\ \mathbf{0} & \mathbf{B}\end{array}\right|$.

We can replace the original problem with an equivalent problem in $R^{(2 r+2)+1}$,

$\min J=x_{0}\left(t_{f}\right)$

subject to

$\dot{\mathbf{y}}=\overline{\mathbf{A}} \mathbf{y}+\overline{\mathbf{B}} \overline{\mathbf{u}}, \mathbf{y}(0)=(0,0,0, \ldots, 0)^{t}$,

$\mathbf{y}\left(t_{f}\right)=\left(x_{0}, \theta, 0, \ldots, 0\right)^{t}$.

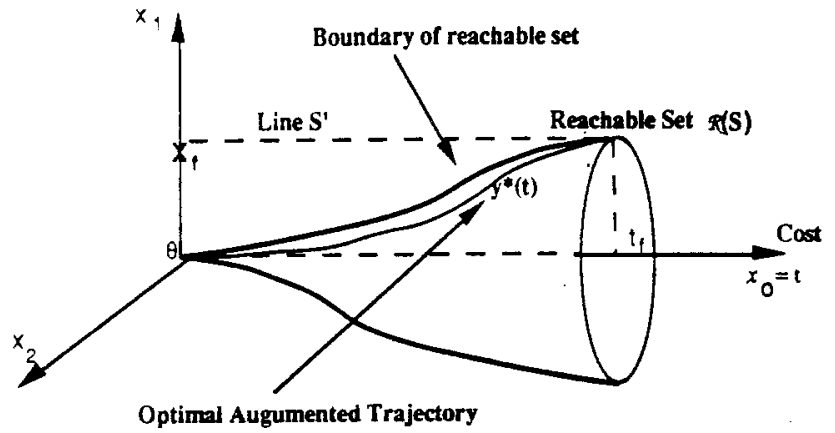

Fig. 11. Illustration of the augmented space

As shown in Fig. 11, the optimal trajectory of (A2) is the curve $\left[\left(x_{0}(t), \mathbf{x}(\mathbf{t})\right]\right.$ within the reachable set which starts from $\left[\begin{array}{ll}0 & x_{0}\end{array}\right]^{t}$ and intersects the line $S^{\prime}$ with minimal $x_{0}$. (The idea of the reachable set will be introduced in detail later in the proof of Theorem 2).

Let us make a small change in the inital condition, $\left[0 \mathbf{x}_{0}^{t}\right]^{t}$, with optimal control $\overline{\mathbf{u}}^{*}(t)$ fixed, and see how this change "propagates" along the optimal trajectory $\mathbf{y}^{*}(t)$. Assume the initial point is perturbed as

$\left[\begin{array}{ll}0 & \mathbf{x}_{0}^{t}\end{array}\right]^{t}+\varepsilon\left[\begin{array}{ll}\gamma_{0} & \gamma^{t}\end{array}\right]^{t}$, where $\varepsilon \ll 1$ and $\left[\gamma_{0} \gamma\right]$ is a vector in
$R^{(2 r+2)+1}$

Let $\psi_{0}$ be $\left[\gamma_{0} \gamma^{t}\right]^{t}$. We find the new trajectory is

$\mathbf{y}^{*}(t)+\varepsilon \psi(t)$

where $\psi(t)$ is described by a homogeneous linear system

$\dot{\psi}(t)=\overline{\mathbf{A}} \psi(t)$ and $\psi(0)=\psi_{0}$

Define the Hamiltonian function of the problem (A2) as: $\mathcal{H}(\mathbf{y}, \overline{\mathbf{p}}, \overline{\mathbf{u}}) \equiv \overline{\mathbf{p}}^{t}\{\overline{\mathbf{A}} \mathbf{y}(t)+\overline{\mathbf{B}} \overline{\mathbf{u}}(t)\}$, where $\overline{\mathbf{p}}=\left[p_{0} \mathbf{p}^{t}\right]^{t}$ is a costate vector of Lagrangian multipliers which satisfies the adjoint differential equation

$\mathrm{d} \overline{\mathbf{p}}(\mathrm{t}) / \mathrm{dt}=-\partial \mathcal{H}^{\mathrm{t}}(\mathbf{y}, \overline{\mathbf{p}}, \overline{\mathbf{u}}) / \partial y=-\overline{\mathbf{A}}^{\mathrm{t}} \overline{\mathbf{p}}$

where $\mathbf{p}$ is the costate vector of the original problem (A1) and $\mathrm{d} p_{0} / \mathrm{dt}=0$. Thus

From (A4) and (A5) we find that $\mathrm{d}\left\{\overline{\mathbf{p}}(t)^{t} \psi(t)\right\} / \mathrm{dt}=0$.

$\left\{\overline{\mathrm{p}}(t)^{t} \psi(t)\right\}=$ constant for the solution of (A4)

with any $\psi_{0}$.

Now if we let $P_{0}$ be a hyperplane passing through $\mathbf{y}_{0}=\left[\begin{array}{ll}0 & \mathbf{x}_{0}\end{array}\right]$ with the equation

$\left[\pi_{0} \pi\right]^{t}\left[\mathbf{y}-\mathbf{y}_{0}\right]=0$

Let $\mathbf{y}-\mathbf{y}_{0}=\psi(0)$ be any vector in $R^{2(r+1)+1}$, and choose $p_{0}=\pi_{0}$ and $\mathbf{p}(0)=\pi$. We have

$\left[\pi_{0} \mathbf{p}(t)\right]^{t}\left[\mathbf{y}(t)-\mathbf{y}^{*}(t)\right]=0$

Thus we can define a hyperplane $P_{t}$ with the equation

$\left[\pi_{0} \mathbf{p}(t)\right]^{t}\left[\mathbf{y}-\mathbf{y}^{*}(t)\right]=0$,

which passes through $\mathbf{y}^{*}(t)$. It is geometrically as a hyperplane moving along the optimal trajectory (as shown in Fig. 12), and we see that our costate will be the normal vector to this particular hyperplane moving along the optimal trajectory.

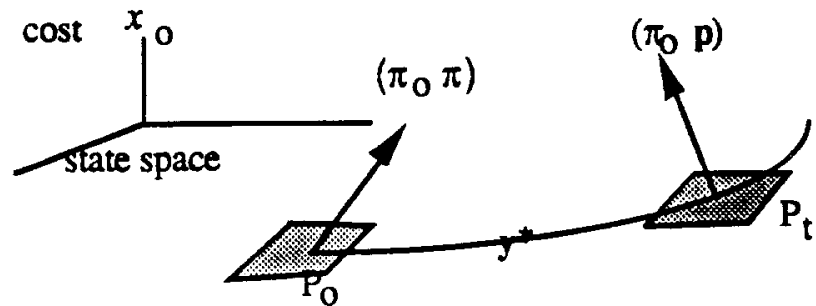

Fig. 12. Illustration of the moving hyperplane $P_{t}$

Finally, since the system (A2) is linear and without state space constraints, we know the optimal control, $\mathbf{u}^{*}(t)$, should be piecewise continuous and of bang-bang type. Thus the optimal trajectory $\mathbf{y}^{*}(t)$ is smooth almost everywhere, except 
at the points where there are discontinuities of control functions. Therefore, the normal vector to the hyperplane moving along the optimal trajectory is unique almost everywhere. Moreover, (A5) is a linear system. We can solve for $\overline{\mathbf{p}}(t)$ analytically in terms of transfer matrix and $\overline{\mathbf{p}}(0)$. If the solution of costates is unique in a finite interval, we can conclude that the costate vector is unique everywhere.

Corollary 3.2.5. Consider a control design model with $r$ flexible modes. Suppose that the number of switching times of the optimal control history between 0 and $t_{f}^{*} / 2$ is $k$, where $r>k$. Then a set of sufficient conditions for optimality are

(i) (14) and the first $k$ equations of (15) admit a solution $\left\{t_{j}, j=1,2, \ldots, k\right.$ and $\left.t_{f}^{*}\right\}$, which is regular;

(ii) $\cos \left(\omega_{i} t_{f}^{*} / 2\right)-2 \cos \left(\omega_{i} t_{k}\right)+2 \cos \left(\omega_{i} t_{k-1}\right)-\ldots+$ $+2(-1)^{k} \cos \left(\omega_{i} t_{1}\right)+(-1)^{k+1}=0$, where $i=k+1, k+$ $2, \ldots, r$;

(iii) $\left\{t_{j}, j=1,2, \ldots, k\right.$ and $\left.t_{f}^{*}\right\}$ satisfy (16) and inequalities (17), with $\left\{p_{0}^{i}=0, i=k+1, k+2, \ldots, r\right\}$.

Proof. It is seen that the first $k+1$ columns of the matrix in (16) form a $(k+1) \times(k+1)$ square matrix which is exactly the transpose of the Jacobian of (14) and the first $k$ equations of (13). Regularity in condition (i) implies that this square coefficient matrix is nonsingular, which implies existence and uniqueness of the solution $\left\{p_{0}^{i}, i=1,2, \ldots, k\right\}$ in (16). Since we have $\left\{p_{0}^{i}=0, i=k+1, k+2, \ldots, r\right\}$ from (iii), it is not difficult to check that conditions (i), (ii) and (iii) indeed solve (14)-(17). Thus the number of switching time of the optimal control history between 0 and $t_{f}^{*} / 2$ is $k, k<r$.

Theorem A2. Suppose the number of flexible modes retained in the control design model is fixed. The optimal maneuver time solved from (14)-(17) is a continuous function of the structural design variables, $\boldsymbol{\xi}$.

Proof. We have the preliminary result that, in general, the mass and stiffness distribution of a spacecraft is a continuous function of structural design parameters. Assume that all eigenvalues derived from (6) and (8) are distinct. Then, the natural frequencies and normalized natural modes of a structure are continuous functions of the structural design parameters. For a time-optimal control problem with a fixed number of flexible modes in the control design model, represented in (12), the configuration of the system equations, all elements in matrices $\mathbf{A}$ and $\mathbf{B}$, which are derived from (11) and (13), are continuous functions of structural design parameters. We represent the matrices as $\mathbf{A}(\xi)$ and $\mathbf{B}(\xi)$ to indicate that they are functions of structural design parameters. Let $\mathbf{A}_{i}=\mathbf{A}\left(\xi_{i}\right)$ and $\mathbf{B}_{i}=\mathbf{B}\left(\xi_{i}\right)$. Thus

$\forall \varepsilon>0, \exists \delta>0$, such that $\forall \xi_{1}, \xi_{2}\left|\xi_{1}-\xi_{2}\right|<\delta$ implies

$\max \left\{\left|\mathbf{A}_{1}-\mathbf{A}_{2}\right|\right\}_{i j}<\varepsilon$ and $\max \left\{\left|\mathbf{B}_{1}-\mathbf{B}_{2}\right|\right\}_{i j}<\varepsilon$.

Let $\mathcal{S}_{i}:\left\{\mathbf{A}_{i}, \mathbf{B}_{i}, \mathbf{U}\right\}$, where $\mathbf{U}$ is the feasible control space which is fixed for any $\xi$, represent a system.
Herein, we only need to prove that $\mathcal{G}$ : the optimal maneuver time of (A1) is a continuous function of the elements in the matrix $\mathbf{A}$ and $\mathbf{B}$ of the system. Let $t_{f i}^{*}$ be the solution of (A1) for a system $\mathcal{S}_{i}$.

We are going to prove $\mathcal{G}$ by making use of the idea of reachable space. Let us first introduce this idea. For a timeoptimal control problem (A1), for a system $\mathcal{S}$, we define the set of reachable states at a time $t$

$\mathcal{R}(\mathcal{S}, t)=$

$=\left\{\mathbf{x}(t) \varepsilon R^{r} ; \mathbf{x}=\mathbf{A x}+\mathbf{B u}, \mathbf{u} \varepsilon \mathbf{U}, \mathbf{x}(0)=\mathbf{0}\right\}$

and the reachable space

$\mathcal{R}(\mathcal{S})=\left\{[\mathbf{x}(t), t] \varepsilon R^{r+1} ; \mathbf{x} \varepsilon \mathcal{R}(\mathcal{S}, t)\right\}$

We claim that the reachable space, $\mathcal{R}(\mathcal{S})$, and the reachable states at time $t, \mathcal{R}(\mathcal{S}, t)$, have the following important properties which will be used to complete the proof.

(i) For any finite time $t, \mathcal{R}(\mathcal{S}, t)$ is a closed set.

Proof. It can be shown that $\forall$ finite time $t, \forall$ a converging sequence $\left\{\mathrm{x}^{i}\right\}$ such that $\forall i, \mathbf{x}^{i} \varepsilon \mathcal{R}\left(\mathcal{S}, t_{1}\right)$ and with the limit $\tilde{\mathbf{x}}$, then $\tilde{\mathbf{x}} \varepsilon \mathcal{R}\left(\mathcal{S}, t_{1}\right)$.

(ii) For any finite time $t, \mathcal{R}(\mathcal{S}, t)$ possesses a boundary. Let $\delta \mathcal{R}(\mathcal{S}, t)$ be the boundary of $\mathcal{R}(\mathcal{S}, t)$.

Proof. For a finite time $t, \mathcal{R}(\mathcal{S}, t)$ is a compact set and every compact subset of $R^{r+1}$ has a boundary.

(iii) For any finite time $t, \mathcal{R}(\mathcal{S}, t)$ is a convex set.

Proof. For any time $t, \forall \mathbf{x}_{1}, \mathbf{x}_{2} \in \mathcal{R}(\mathcal{S}, t)$ and driven by the control $\mathbf{u}_{1}, \mathbf{u}_{\mathbf{2}}$, respectively, we have

$\mathbf{x}_{\mathbf{3}}=\lambda \mathbf{x}_{1}+(1-\lambda) \mathbf{x}_{\mathbf{2}}, \quad 0 \leq \lambda \leq 1$,

can also be reached at time $t$.

(iv) For any finite time $t, \mathcal{R}(\mathcal{S}, t)$ is nondecreasing in the sense that

$\forall t_{1}$ and $t_{2}$ if $t_{1}<t_{2}$, then $\mathcal{R}\left(\mathcal{S}, t_{1}\right) \subseteq \mathcal{R}\left(\mathcal{S}, t_{2}\right)$

Proof. Consider (A3). We know that $\forall \mathbf{x}_{1} \varepsilon \mathcal{R}\left(\mathcal{S}, t_{1}\right), \mathbf{x}_{1}$ can also be reached at time $t_{2}$ with the control $\mathbf{u}=0$ for $t<t_{2}-t_{1}$ and $\mathbf{u}=\mathbf{u}_{1}\left[t-\left(t_{2}-t_{1}\right)\right]$ otherwise, where is $u_{1}$ the same control driving the system to $x_{1}$.

(v) From Fig. 13, it is clear that the optimal maneuver time is the time when the final state first penetrates the boundary of the reachable space. Thus

$t_{f}^{*}=\min _{t}\left\{\mathbf{x}_{\mathbf{f}} \in \mathcal{R}(\mathcal{S}, t)\right\}=\min _{t}\left\{\mathbf{x}_{\mathbf{f}} \in \delta \mathcal{R}(\mathcal{S}, t)\right\}$ 


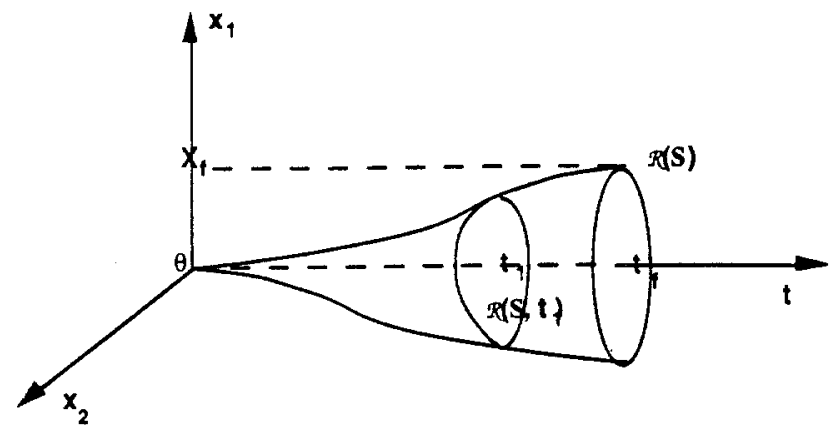

Fig. 13. The reachable space $\mathcal{R}(\mathcal{S})$ and the set of reachable states at a specific time $t_{1}, \mathcal{R}\left(\mathcal{S}, t_{1}\right)$

Now we prove $\mathcal{G}$ by contradiction.

Suppose $\exists \varepsilon>0$, such that

$\forall \delta>0, \exists \mathbf{A}_{1}, \mathbf{A}_{2}, \mathbf{B}_{1}$, and $\mathbf{B}_{2}$ satisfying

$\max \left\{\left|\mathbf{A}_{1}-\mathbf{A}_{2}\right|\right\}_{i j}<\delta$, and

$\max \left\{\left|\mathbf{B}_{1}-\mathbf{B}_{2}\right|\right\}_{i j}<\delta$, and where $\left|t_{f 1}^{*}-t_{f 2}^{*}\right| \geq \varepsilon$.

Since $\left|t_{f 1}^{*}-t_{f 2}^{*}\right| \geq \varepsilon$, without loss of generality, suppose $t_{f 2}^{*}>t_{f 1}^{*}$.

From (A6), we have at time $t_{f 1}^{*}, \mathbf{x}_{\mathrm{f}} \in \delta \mathcal{R}\left(\mathcal{S}_{2}, t\right)$, but $\mathbf{x}_{\mathbf{f}} \notin \delta \mathcal{R}\left(\mathcal{S}_{2}, t\right)$. This is because if $\mathbf{x}_{\mathbf{f}} \in \delta \mathcal{R}\left(\mathcal{S}_{2}, t\right)$, we have $t_{f 2}^{*}<t_{f 1}^{*}$.

Thus we have:

$\min \left\|\mathbf{x}-\mathbf{x}_{\mathbf{f}}\right\| \geq \rho, \rho>0, \mathbf{x}_{\mathbf{f}} \in \mathcal{R}\left(\mathcal{S}_{2}, t\right)$

Let $\mathbf{X}$ be the point which is nearest to $\mathbf{x}_{\mathbf{f}}$ and belong to $\mathcal{R}\left(\mathcal{S}_{2}, t\right)$. Suppose that for the system $\mathcal{S}_{1}, \mathbf{x}_{\mathbf{f}}$ is driven by the control $\mathbf{u}_{1}(\tau), 0 \leq \tau \leq t_{f 1}^{*}$. Suppose that for the system $\mathcal{S}_{2}, \mathbf{Y}$ is the state driven by $\mathbf{u}_{1}$.

Therefore we have

$\left\|\mathbf{X}-\mathbf{x}_{\mathbf{f}}\right\| \leq\left\|\mathbf{Y}-\mathbf{x}_{\mathbf{f}}\right\|$

However, we know $\mathbf{x}(t)=\int_{0}^{t} e^{A(t-\tau)} B u(\tau) \mathrm{d} \tau$. For fixed $u$ and $t, \mathbf{x}(t)$ is a $C^{1}$ function of $\mathbf{A}$ and $\mathbf{B}$. Thus, $\forall \varepsilon^{\prime}>0, \exists \delta>0$, such that when $\max \left\{\left|\mathbf{A}_{1}-\mathbf{A}_{2}\right|\right\}_{i j}<\delta$, and $\max \left\{\left|\mathbf{B}_{1}-\mathbf{B}_{2}\right|\right\}_{i j}<\delta$, we have $\left\|\mathbf{Y}-\mathbf{x}_{\mathbf{f}}\right\| \leq \varepsilon^{\prime}$. (A18) Now, let $\varepsilon^{\prime}=\varepsilon / 2$, and from (A17) and (A18) we have

$\varepsilon \leq\left\|\mathbf{X}-\mathbf{x}_{\mathbf{f}}\right\| \leq\left\|\mathbf{Y}-\mathbf{x}_{\mathbf{f}}\right\| \leq \varepsilon / 2, \varepsilon>0$.

This contradiction completes the proof of statement $\mathcal{G}$.

Theorem A3. Consider the general design case where the structural properties are analytical functions of the design parameters. Suppose the number of flexible modes retained in the model is fixed, say $r$. Assume that in a closed region of the feasible design variable space, the number of switching times, say $k$, follows the generic rule, where $k=r$. Then the optimal maneuver time $t_{f}^{*}$ is an analytical function of the structural design variables $\xi$, in this region.

Proof. First, it is not difficult to see that the $k+1$ (equal to $r+1$ by assumption) equations of (14) and (15) together admit many solutions $\left\{t_{j}, j=1,2, \ldots k\right.$ and $\left.t_{f}^{*}\right\}$, which are also regular. We only concern ourselves with the solution manifolds of (14) and (15) which are regular. Since the solution of the time optimal control problem is unique, only one of them will satisfy all necessary conditions of Corollary 3.2.4. We rewrite (16) and (17)

$[\mathbf{\Phi}]\{\mathbf{p}\}=\{\mathbf{c}\}$

where $[\boldsymbol{\Phi}],\{\mathbf{p}\},\{\mathbf{c}\}$ are defined in Section 3 .

$t_{f}^{*} / 2>t_{k}>t_{k-1}>\ldots>t_{2}>t_{1}>0$

$p_{0}^{0} t+\sum_{i=1}^{r} \beta_{j}^{i} p_{0}^{i} \sin \left(\omega_{i} t\right) \neq 0$,

where $0 \leq t \leq t_{f}^{*} / 2, \quad t \neq t_{i}, \quad i=1,2, \ldots, k$.

The solutions of (14) and (15) are regular, and the coefficient matrix $[\Phi]$ in (16) is the transpose of the Jacobian of

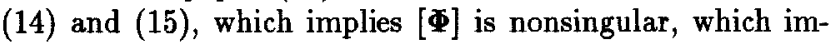
plies we can solve for a unique $\{\mathbf{p}\}$, hence (16) can be satisfied. Here, we only need to show that the solution set of $\left\{t_{j}, j=1,2, \ldots k\right.$ and $\left.t_{f}^{*}\right\}$ which further satisfy (17a) and (17b), will not jump along the solution branches of (14) and (15) when we perturb the design variables; then, from the implicit function theorem (see, e.g. Hartman 1982), $t_{f}^{*}$ is an analytical function. Since (14) and (15) are regular, solutions of $\left\{t_{j}, j=1,2, \ldots k\right.$ and $\left.t_{f}^{*}\right\}$ are analytical functions of the design parameters. It is evident that $\left\{t_{j}, j=1,2, \ldots k\right.$ and $\left.t_{f}^{*}\right\}$ will still satisfy (17a) when we perturb design parameters. Since when we differentiate both sides of (16) with respect to a design parameter $\xi$, we have

$\{\partial[\mathbf{\Phi}] / \partial \xi\}\{\mathbf{p}\}+[\mathbf{\Phi}]\{\partial \mathbf{p} / \partial \xi\}=\{\partial \mathbf{c} / \partial \xi\}$

$\{\partial \mathbf{p} / \partial \xi\}=[\Phi]^{-1}[\{\partial \mathbf{c} / \partial \xi\}-\{\partial[\mathbf{\Phi}] / \partial \xi\}\{\mathbf{p}\}]$

where $\partial[\Phi] / \partial \xi$ represents $\left\{\partial \phi_{i j} / \partial \xi, i=1,2, \ldots, k+1(r\right.$ is equal to $k), \phi_{i j}$ are the entries of $\left.[\Phi]\right\},\{\partial p / \partial \xi\}$ represents $\left\{\partial p_{i} / \partial \xi, i=1,2, \ldots, r+1, p_{i}\right.$ are the entries of $\left.\mathbf{p}\right\}$, and $\{\partial c / \partial \xi\}$ represents $\left\{\partial c_{i} / \partial \xi, i=1,2, \ldots, r+1, c_{i}\right.$ are the entries of $\mathbf{c}\}$.

Because $[\boldsymbol{\Phi}]$ is nonsingular and analytical and $\{\partial \mathbf{c} / \partial \xi\}$ is analytical, we know $\{\partial \mathbf{p} / \partial \xi\}$ exist, which implies $\{\mathbf{p}\}$ is a continuous function of $\xi$, hence all parameters in $(17 \mathrm{~b})$ are continuous functions of $\xi$. Thus $\left\{t_{j}, j=1,2, \ldots k\right.$ and $\left.t_{f}^{*}\right\}$ will satisfy (17b) when we perturb the design parameters. 